\title{
Asymmetric Organocatalytic Synthesis of $\beta$-Hydroxyynones with a Quaternary Carbon Center under Aqueous Conditions
}

\author{
Guowei Kang, Jun Jiang, * Hongxin Liu and Huayue Wu* \\ College of Chemistry and Materials Engineering, Wenzhou University, Chashan University Town, \\ Wenzhou, Zhejiang Province 325035, People's Republic of China
}

\begin{abstract}
A reação aldólica direta de metil inonas não-modificadas sob condições aquosas catalisada pela amina terciária quiral de tiouréia é descrita. Este procedimento evitou a reação retroaldólica dos produtos $\beta$-hidroxinonas, e admite as isatinas (1H-indol-2,3-diona) e os menos ativos ésteres $\alpha$-ceto acíclicos como aceptores, oferecendo um arranjo estruturalmente diverso de $\beta$-hidroxinonas tendo um centro de carbono quaternário, com rendimentos de moderado a bom e enancioseletividade.
\end{abstract}

The chiral tertiary amine thiourea catalyzed direct aldol reaction of unmodified methyl ynones under aqueous conditions is described. This procedure avoided the retro-aldol reaction of the $\beta$-hydroxyynone products, and tolerated both the isatin (1H-indole-2,3-dione) and the less active acyclic $\alpha$-keto esters as acceptors, affording a structurally diverse array of $\beta$-hydroxyynones bearing a quaternary carbon center with moderate to good yields and enantioselectivities.

Keywords: $\beta$-hydroxyynones, chiral thiourea, aldol reaction, aqueous condition

\section{Introduction}

Enantioriched $\beta$-hydroxyynones are highly functionalized building blocks for the synthesis of biologically active compounds and natural products, such as fostriecin ${ }^{1}$ and maitotoxin. ${ }^{2}$ Although the aldol reaction is the most efficient way to construct $\beta$-hydroxy carbonyl structures, the $\beta$-hydroxyynone products are usually synthesized via the addition of alkynyllithium or alkynylmagnesium halide to the corresponding $\beta$-hydroxylated Weinreb amides. ${ }^{3-12}$ This is due to the fact that ynones are good Michael acceptors and the ynone-derived $\beta$-hydroxy ketones tend to undergo retro-aldol reactions. ${ }^{13,14}$ Thus, direct catalytic asymmetric aldol reactions involving ynones are highly desirable. In 2004, Trost et al. ${ }^{13}$ reported the first enantioselective aldol additions of methyl ynones to $\alpha$-ketal aldehydes using a chiral dinuclear zinc catalyst. Subsequently, Silva et al. ${ }^{14}$ found that proline derived sulfonamide could efficiently catalyze the aldol reaction of the methoxymethyl ether (MOM)-protected ynones and aldehyde with good yields and enantioselectivities. ${ }^{14}$ However, the unmodified simple methyl ynones are not good substrates under proline-sulfonamide catalysis conditions with low yields because of several

*e-mail: junjiang@wzu.edu.cn; huayuewu@wzu.edu.cn side products. ${ }^{14}$ More importantly, no example of the asymmetric catalytic synthesis of $\beta$-hydroxyynones resulting in a quaternary carbon center has been reported. Since List et al. ${ }^{15}$ reported the proline catalyzed cross-aldol reaction between ketones and aldehydes, ${ }^{15}$ many amine derivatives such as proline-amide type catalysts ${ }^{16-18}$ have been developed for such transformations, ${ }^{19-22}$ being most of which based on the enamine-activiation mechanism. ${ }^{19-22}$ However, in comparison with simple ketones, the formation of enamine intermediates with primary or secondary amine catalysts and inactive unmodified ynones remains a great challenge. Based on the fact that DABCO can efficiently catalyze the direct aldol reaction between simple methyl ynone and isatin (1H-indole-2,3-dione) in our pilot experiment, it was envisioned the developing of the chiral tertiary amine catalysts in order to realize this asymmetric transformation. During the preparation of this study, Guo et $a l .{ }^{23}$ and Allu et al. ${ }^{24}$ reported chiral tertiary amine thiourea and urea catalyzed enantioselective aldol reactions of ketones with excellent results. Herein, we report our preliminary results with chiral tertiary amine thiourea ${ }^{25}$ catalyzed direct aldol reactions of methyl ynones, affording $\beta$-hydroxyynones with a quaternary carbon center under aqueous conditions ${ }^{26}$ with moderate to good yields and enantioselectivites (Figure 1). 


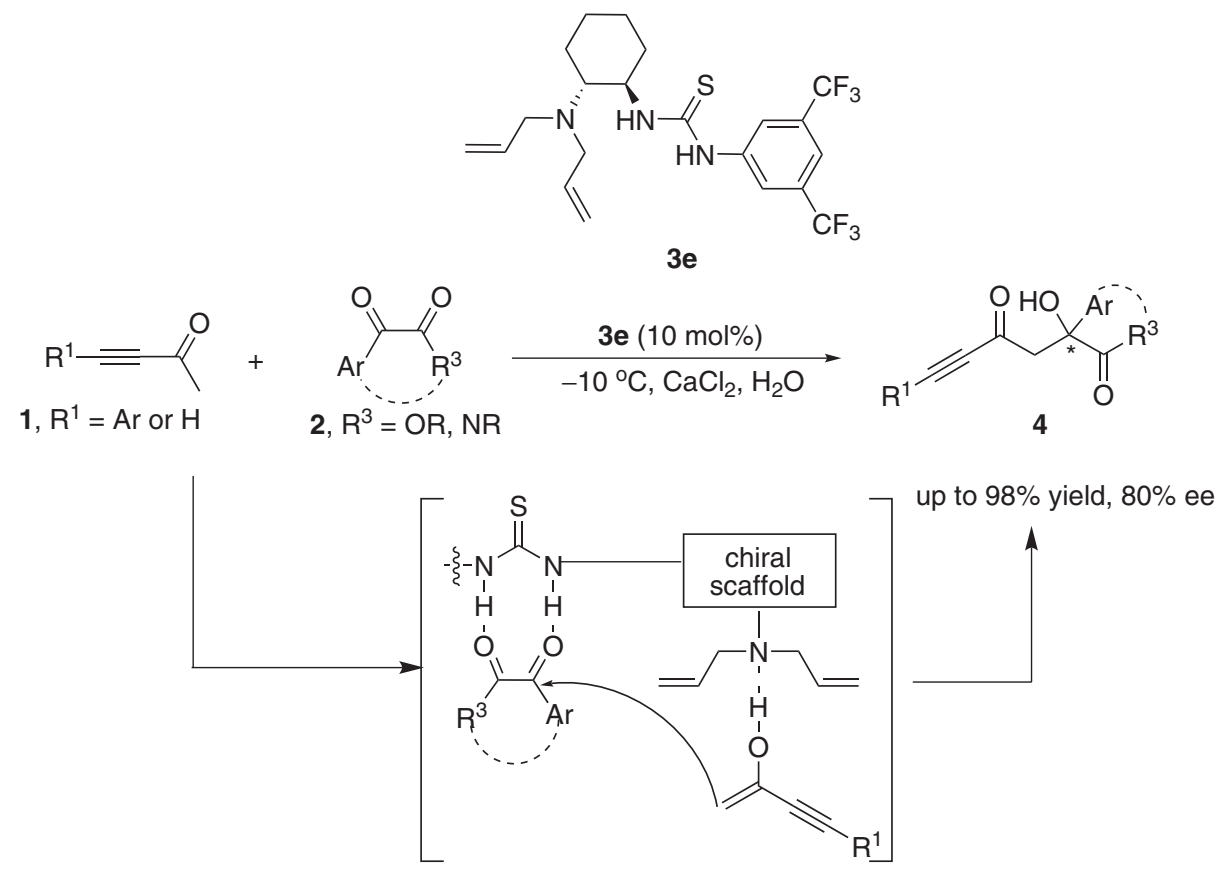

Figure 1. Thiourea catalyzed direct aldol reaction of methyl ynone and active ketones.

\section{Results and Discussion}

The organocatalyzed asymmetric aldol reactions between simple ketones or aldehydes and isatins have been intensely studied, ${ }^{27-37}$ as these chiral 3-alkyl3-hydroxyindolin-2-one products are important building blocks in both natural products and medicinal compound syntheses. ${ }^{38,39}$ However, the asymmetric aldol reactions between ynones and isatins remain a synthetic challenge since the products tend to undergo retro-aldol reaction in organic solvents. For example, $42 \%$ of product $4 \mathbf{a}$ converted to starting materials after stirring with $20 \mathrm{~mol} \%$ DABCO in $\mathrm{CH}_{2} \mathrm{Cl}_{2}$ for $24 \mathrm{~h}$, and a similar result was obtained when $\mathrm{CHCl}_{3}$ was used as solvent. Moreover, ynones have the ability to act as both electrophile and nucleophile under certain conditions, such as base catalysis. Initially, our investigation began with the reaction of ynone 1a with $\mathrm{N}$-butyl substituted isatin 2a by using $20 \mathrm{~mol} \%$ of DABCO as the catalyst. To our delight, the reaction catalyzed by DABCO gave the desired product with $80 \%$ yield in 3 days at room temperature in $\mathrm{CHCl}_{3}$. Several solvents were then tested (Table 1, entries 1-6), and water was found to dramatically enhance the reaction rate with $88 \%$ yield in $1 \mathrm{~h}$ (Table 1, entry 6). Because this $\beta$-hydroxyynone product was stable in water, it was supposed that using water as the solvent may not only increase the reaction concentration but also avoid the retro-aldol reaction.

In the sequence, it was examined the asymmetric catalytic ability of chiral thiourea catalysts (Figure 2) at $0{ }^{\circ} \mathrm{C}$ with 5 equivalents of ynone 1a, using brine as solvent instead of water to avoid freezing (Table 1, entries 7-13). The reactions catalyzed by quinine and cinchonidine proceeded smoothly but with very low enantioselectivities (ee) (Table 1, entries 7-8). Better results were obtained when tertiary amine thioureas were used as catalysts (Table 1 , entries 9-13). The screening studies revealed that catalyst 3e was the best choice in terms of enantioselectivity, which gave the desired product with $82 \%$ yield and $71 \%$ ee after $10 \mathrm{~h}$. The variation of the group bonded to nitrogen of the isatin heterocycle did not increase the enantioselectivity (Table 1, entries 13-17). For example, $N$-hexyl isatin or $\mathrm{N}$-phenyl isatin led to slightly lower enantioselectivities (Table 1, entries 16-17), while the use of $N$-methyl isatin afforded the corresponding product 4ab in relatively low ee (Table 1, entry 14), and $N$-Boc isatin was found inactive when used in this reaction under aqueous condition. Next, several solvents were screened at $0{ }^{\circ} \mathrm{C}$ with 5 equivalents of ynone 1a to improve the enantioselectivity (Table 2). The reactions in these screened organic solvents either led to long reaction time and low yield or decreased the enantiocontrol (Table 2, entries1-5). Further experiments investigated the influence of additives. It was shown that salts affect the enantioselectivity. The best result was obtained when $\mathrm{CaCl}_{2}$ was used as the additive, gaving the desired product with $93 \%$ yield and $73 \%$ ee at $0{ }^{\circ} \mathrm{C}$. Finally, the enantioselectivity was improved to $80 \%$ ee by cooling the reaction to $-10{ }^{\circ} \mathrm{C}$. Further cooling $\left(-20^{\circ} \mathrm{C}\right)$ was tried, but did not improve the enantioselectivity. The reaction was then performed on a $1 \mathrm{mmol}$ scale of $\mathbf{2 a}$ and $5 \mathrm{mmol}$ of $\mathbf{1 a}$ in $5 \mathrm{~mL}$ of solvent under the optimal conditions (Table 2, entry 13). 
Table 1. Catalyst screening on the direct aldol reaction between ynone and isatin ${ }^{\mathrm{a}}$

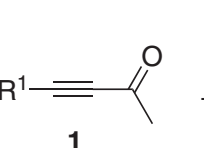

1<smiles>O=C1Nc2ccccc2C1=O</smiles>

$2 R^{2}$

2a: $R^{2}=n-B u t y l$

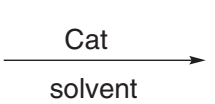

solven

(2)

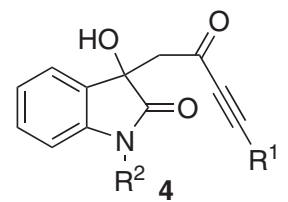

$$
\begin{array}{ll}
\text { 1b: } R^{1}=4-M e-\mathrm{C}_{6} \mathrm{H}_{4} & \text { 2ab: } R^{2}=\mathrm{Me} \\
\text { 1c: } R^{1}=\mathrm{H} & \text { 2ac: } R^{2}=A l l y l \\
& \text { 2ad: } R^{2}=\mathrm{n}-\mathrm{C}_{6} \mathrm{H}_{13} \\
& \text { 2ae: } R^{2}=\mathrm{Ph}
\end{array}
$$

\begin{tabular}{|c|c|c|c|c|c|c|}
\hline entry & Catalyst & 2 & Solvent & 4 & Yield / \% ${ }^{\mathrm{d}}$ & ee $/ \%^{\circ}$ \\
\hline $1^{\mathrm{b}}$ & DABCO & $2 a$ & dioxane & $4 a$ & 24 & - \\
\hline $2^{\mathrm{b}}$ & DABCO & $\mathbf{2 a}$ & $\mathrm{CH}_{2} \mathrm{Cl}_{2}$ & $4 a$ & 18 & - \\
\hline $3^{\mathrm{b}}$ & DABCO & $2 a$ & $\mathrm{CHCl}_{3}$ & $4 \mathbf{a}$ & 10 & - \\
\hline $4^{b}$ & DABCO & $2 \mathbf{a}$ & $\mathrm{THF}$ & $4 \mathbf{a}$ & 19 & - \\
\hline $5^{b}$ & $\mathrm{DABCO}$ & $2 \mathbf{a}$ & toluene & $4 a$ & 21 & - \\
\hline $6^{\mathrm{b}}$ & DABCO & $\mathbf{2 a}$ & $\mathrm{H}_{2} \mathrm{O}$ & $4 a$ & 88 & - \\
\hline $7^{\mathrm{c}}$ & quinine & $2 \mathbf{a}$ & brine & $4 \mathbf{a}$ & 91 & 6 \\
\hline $8^{c}$ & cinchonidine & $2 \mathbf{a}$ & brine & $4 a$ & 87 & 12 \\
\hline 9 & $3 \mathbf{a}$ & $2 \mathbf{a}$ & brine & $4 \mathbf{a}$ & 89 & 38 \\
\hline 10 & $3 \mathbf{b}$ & $2 \mathbf{a}$ & brine & $4 a$ & 92 & 56 \\
\hline 11 & $3 c$ & $2 \mathbf{a}$ & brine & $4 a$ & 95 & 50 \\
\hline 12 & $3 d$ & $2 \mathbf{a}$ & brine & $4 a$ & 96 & 45 \\
\hline 13 & $3 e$ & $2 \mathbf{a}$ & brine & $4 a$ & 82 & 71 \\
\hline 14 & $3 \mathbf{e}$ & $2 a b$ & brine & $4 a b$ & 98 & 56 \\
\hline 15 & $3 e$ & $2 a c$ & brine & $4 a c$ & 95 & 65 \\
\hline 16 & $3 \mathbf{e}$ & $2 \mathrm{ad}$ & brine & 4ad & 91 & 69 \\
\hline 17 & $3 e$ & $2 \mathrm{ae}$ & brine & $4 a e$ & 97 & 68 \\
\hline
\end{tabular}

4a: $R^{1}=P h, R^{2}=n-B u t y l$

4ab: $R^{1}=P h, R^{2}=M e$

4ac: $R^{1}=P h, R^{2}=$ Allyl

4ad: $\mathrm{R}^{1}=\mathrm{Ph}, \mathrm{R}^{2}=\mathrm{n}-\mathrm{C}_{6} \mathrm{H}_{13}$

2ae: $\mathrm{R}^{1}=\mathrm{Ph}, \mathrm{R}^{2}=\mathrm{Ph}$

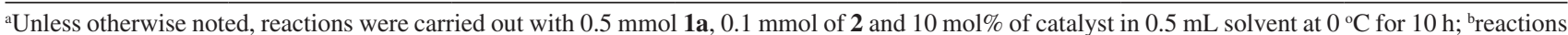

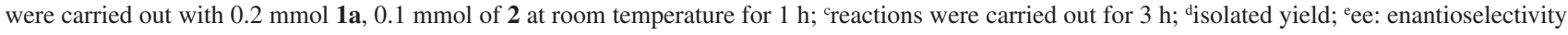
determined by chiral HPLC.<smiles>COc1ccc2nccc(C(NC(=S)Nc3cc(C(F)(F)F)cc(C(F)(F)F)c3)N3CC4CCC3CC4)c2c1</smiles>

$3 a$<smiles>CN(C)C1CCCCC1NC(=S)Nc1cc(C(F)(F)F)cc(C(F)(F)F)c1</smiles>

3b<smiles>CCON(CC)C1CCCCC1NC(=S)Nc1cc(C(F)(F)F)cc(C(F)(F)F)c1</smiles>

3d<smiles>CCCCCN(CCCCC)C1CCCCC1NC(=S)Nc1cc(C(F)(F)F)cc(C(F)(F)F)c1</smiles>

$3 c$<smiles>C=CCN(CC=C)[C@@H]1CCCC[C@H]1NC(=S)Nc1cc(C(F)(F)F)cc(C(F)(F)F)c1</smiles>

$3 e$

Figure 2. Chemical structure of screened catalysts.

However, decreased enantioselectivity was observed (85\% yield, 53\% ee).

With the optimal reaction conditions in hand, it was then examined the scope of the direct aldol reactions between ynones and active ketones. The reactions were generally conducted in saturated $\mathrm{CaCl}_{2}$ solution at -10 to $20{ }^{\circ} \mathrm{C}$ for $9-72 \mathrm{~h}$. The results are summarized in Table 3. All of the isatins showed high reactivities and 
Table 2. Effects of reaction condition on the direct aldol reaction between ynone and isatin ${ }^{\mathrm{a}}$

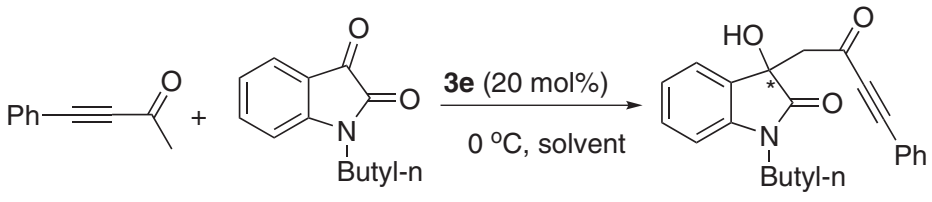

$1 a \quad 2 a$

$4 a$

\begin{tabular}{|c|c|c|c|c|c|}
\hline entry & Solvent & time $/ \mathrm{h}$ & Temperature $/{ }^{\circ} \mathrm{C}$ & Yield / \%b & ee $/ \%^{\mathrm{c}}$ \\
\hline 1 & $\mathrm{CHCl}_{3}$ & 48 & 0 & 77 & 59 \\
\hline 2 & Toluene & 48 & 0 & 90 & 57 \\
\hline 3 & THF & 48 & 0 & 25 & 66 \\
\hline 4 & $\mathrm{CH}_{3} \mathrm{CN}$ & 48 & 0 & 66 & 64 \\
\hline 5 & $\mathrm{MeOH}$ & 48 & 0 & 80 & 24 \\
\hline 6 & $\mathrm{AcONa} / \mathrm{H}_{2} \mathrm{O}$ & 9 & 0 & 96 & 68 \\
\hline 7 & $\mathrm{KF} / \mathrm{H}_{2} \mathrm{O}$ & 9 & 0 & 62 & 58 \\
\hline 8 & $\mathrm{NH}_{4} \mathrm{Cl} / \mathrm{H}_{2} \mathrm{O}$ & 9 & 0 & 97 & 65 \\
\hline 9 & $\mathrm{LiF} / \mathrm{H}_{2} \mathrm{O}$ & 9 & 0 & 97 & 66 \\
\hline 10 & $\mathrm{KBr} / \mathrm{H}_{2} \mathrm{O}$ & 9 & 0 & 97 & 67 \\
\hline 11 & $\mathrm{CaCl}_{2} / \mathrm{H}_{2} \mathrm{O}$ & 9 & 0 & 93 & 73 \\
\hline 12 & $\mathrm{CaCl}_{2} / \mathrm{H}_{2} \mathrm{O}$ & 14 & -10 & 95 & 80 \\
\hline $13^{\mathrm{d}}$ & $\mathrm{CaCl}_{2} / \mathrm{H}_{2} \mathrm{O}$ & 12 & -10 & 85 & 53 \\
\hline
\end{tabular}

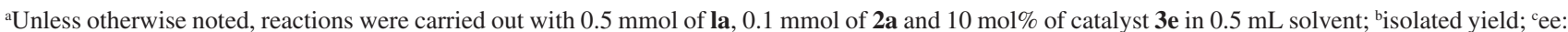
enantioselectivity determined by chiral HPLC; ${ }^{\mathrm{d}}$ reaction was performed on a $1 \mathrm{mmol}$ scale of $\mathbf{2 a}$ and $5 \mathrm{mmol}$ of $\mathbf{1 a}$ in $5 \mathrm{~mL}$ solvent.

afforded the $\beta$-hydroxyynones containing a quaternary carbon center with excellent yields and moderate to good ees. The position of the substituent on the isatin had an obvious influence on the enantioselectivity. For example, 4 -substituted isatins afforded products with low ees (Table 3, entries 2-3, 46-54\% ee), while substituents at other positions on the isatin heterocycle led to higher enantioselectivities (Table 3, entries 4-11, 57-78\% ee). The electronic nature of the substituents also plays an important role on the enantiocontrol. Isatins with an electron-donating group on the aromatic ring gave similar results to $2 \mathbf{a}$ (Table 3 , entries 1, 4 and 9), whilst the presence of an electron-withdrawing group resulted in reduced enantioselectivity (such as Table 3, entry 8). Next, other ynones were also tested. Because both of the reactants are solid, the reaction involving $\mathbf{1 b}$ was carried out in $\mathrm{CH}_{3} \mathrm{CN}$ instead of $\mathrm{CaCl}_{2}$ solution, and led to the product $4 \mathbf{b a}$ with $53 \%$ yield and $78 \%$ ee (Table 3, entry 12 ). When but-3-yn-2-one $1 \mathbf{c}$ was used as the donor in this reaction, the racemic product was obtained (Table 3, entry 13). Notably, besides isatins, less active acyclic $\alpha$-keto esters $\mathbf{2 l - 2 0}$ can also be applied in this reaction, but only under aqueous conditions. The reaction of $\alpha$-keto esters with ynone 1a led to the desired products with moderate yields and enantioselectivities (Table 3, entries $14-17,34-44 \%$ yield, $47-63 \%$ ee). The ester group will affect the yield and ee as the result of steric hindrance. For example, more sterically congested $\mathbf{2 m}$ and $\mathbf{2 n}$ afforded the $\beta$-hydroxyynones with relatively low yields and moderate ees (Table 3, entries 15-16).

\section{Conclusion}

In conclusion, we have developed the chiral tertiary amine thiourea catalyzed direct aldol reactions of unactivated methyl ynones, efficiently resulting in $\beta$-hydroxyynones containing a quaternary carbon center as the only product with moderate to good yields and enantioselectivities (34-98\% yield, 46-80\% ee). Importantly, aqueous conditions were found to not only dramatically enhancing the reaction rate, but also increase the enantioselectivity. Notably, besides isatins, less active acyclic $\alpha$-keto esters can also be used as substrates in this reaction under standard conditions, albeit in lower yields.

\section{Experimental}

Nuclear magnetic resonance (NMR) spectra were recorded on a Bruker-500 $\mathrm{MHz}$ spectrometer. High-resolution mass spectrometry (HRMS, Micromass GCT-MS) spectra were recorded on a P-SIMS-Gly from 
Table 3. Direct aldol reaction of ynones and active ketones ${ }^{\mathrm{a}}$

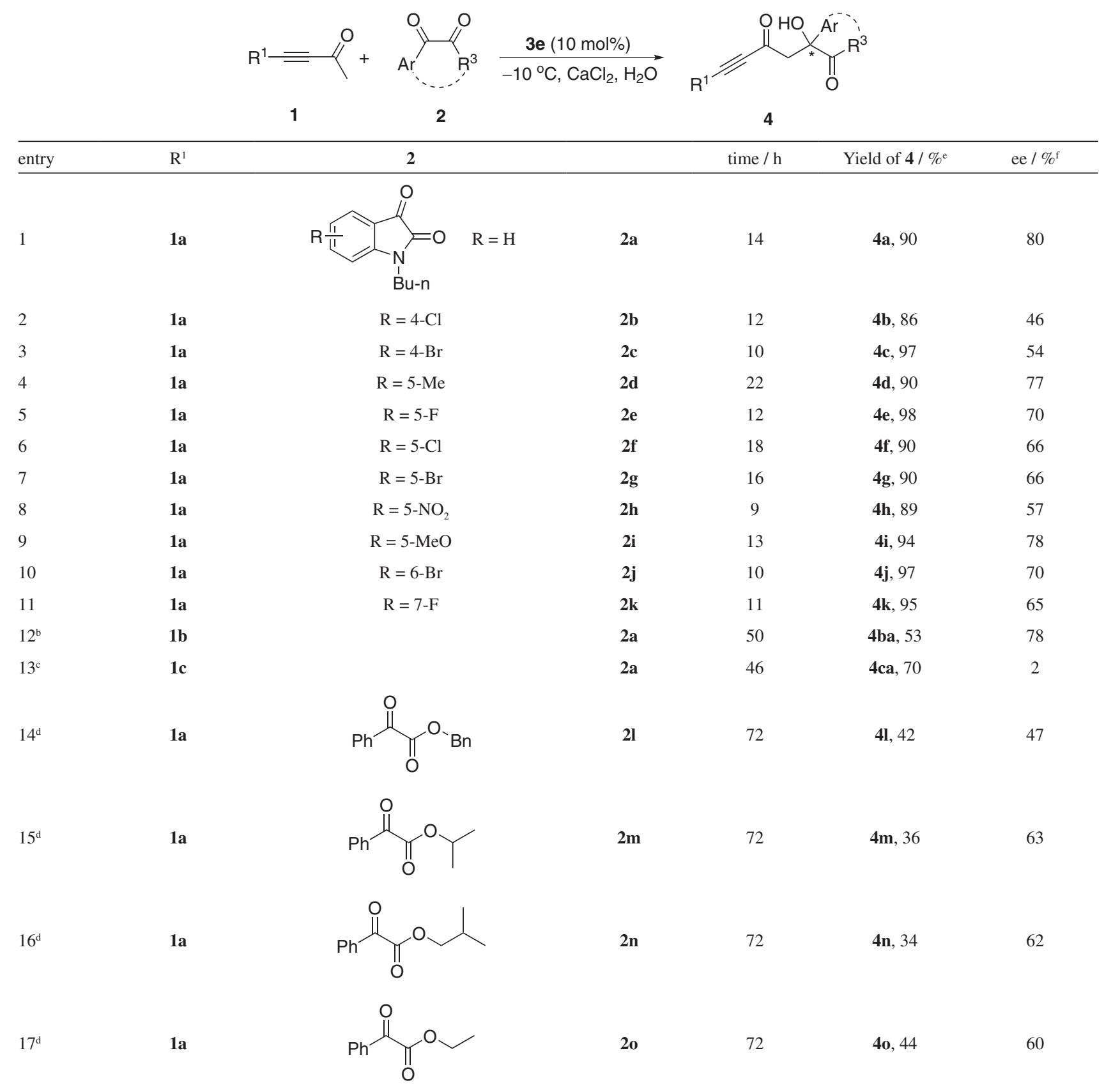

anless otherwise noted, reactions were carried out with $0.5 \mathrm{mmol}$ of $\mathbf{1}, 0.1 \mathrm{mmol}$ of 2 and $10 \mathrm{~mol} \%$ of catalyst $3 \mathrm{e}$ at $-10{ }^{\circ} \mathrm{C}$ in $0.5 \mathrm{~mL}$ saturated $\mathrm{CaCl}_{2}$ solution; ${ }^{\mathrm{b}}$ reaction was carried out in $\mathrm{CH}_{3} \mathrm{CN}$ at $-5{ }^{\circ} \mathrm{C}$; ${ }^{\mathrm{c}}$ reaction was carried out at $10{ }^{\circ} \mathrm{C}$; ${ }^{\mathrm{d}}$ reaction was carried out at $20{ }^{\circ} \mathrm{C}$; ${ }^{\mathrm{e}}$ isolated yield; ${ }^{\mathrm{f}} \mathrm{ee}$ : enantioselectivity determined by chiral HPLC.

Bruker Daltonics Inc. Infrared (IR) spectra were recorded on a Nicolet MX-1E FT-IR spectrometer. High performance liquid chromatography (HPLC) analysis was performed on a Shimadzu LC-20AT series HPLC. Chiralpak AS, $\mathrm{AD}, \mathrm{OD}$ and IA were purchased from Daicel Chemical Industries, Ltd. Optical rotation data were acquired on a PolAAr 3005 automatic polarimeter. Solvents for the column chromatography were distilled before use.
General procedure for the asymmetric organocatalytic synthesis of $\beta$-hydroxyynones with a quaternary carbon center under aqueous conditions

Active ketone $(0.1 \mathrm{mmol})$ and catalyst $\mathbf{3 e}(10 \mathrm{~mol} \%)$ were added to the saturated $\mathrm{CaCl}_{2}$ solution $(0.5 \mathrm{~mL})$ in a tube and the mixture was stirred at the corresponding temperature for $15 \mathrm{~min}$, then the ynone $(0.5 \mathrm{mmol})$ was 
added and the resulting mixture was stirred for 9-72 $\mathrm{h}$ at the determined temperature (monitored by thin layer chromatography (TLC)). The reaction mixture was extracted with $\mathrm{CH}_{2} \mathrm{Cl}_{2}(3 \times 0.5 \mathrm{~mL})$ and the organic phase was purified by flash column chromatography on silica gel (eluent: petroleum ether/ethyl acetate $=5 / 1-3 / 1$ ) to yield pure products.

\section{Supplementary Information}

Supplementary data are available free of charge at http://jbcs.sbq.org.br as a PDF file.

\section{Acknowledgements}

We are grateful for the financial support from NSFC (21002071 and 21072153), Department of Education of Zhejiang Province (Y201017083) and Science and Technology Department of Zhejiang Province (2009R50002).

\section{References}

1. Trost, B. M.; Frederiksen, M. U.; Papillon, J. P. N.; Harrington, P. E.; Shin, S.; Shireman, B. T.; J. Am. Chem. Soc. 2005, 127, 3666.

2. Nicolaou, K. C.; Aversa, R. J.; Jin, J.; Rivas F.; J. Am. Chem. Soc. 2010, 132, 6855.

3. Chavez, D. E.; Jacobsen, E. N.; Angew. Chem., Int. Ed. 2001, 40, 3667.

4. Marshall, J. A.; Ellis, K.; Tetrahedron Lett. 2004, 45, 1351.

5. Evans, D. A.; Starr, J. T.; J. Am. Chem. Soc. 2003, 125, 13531.

6. Fujii, K.; Maki, K.; Kanai, M.; Shibasaki, M.; Org. Lett. 2003, 5,733 .

7. Funel, J-A.; Prunet, J.; J. Org. Chem. 2004, 69, 4555.

8. Shin, Y.; Fournier, J.-H.; Balachandran, R.; Madiraju, C.; Raccor, B. S.; Zhu, G.; Edler, M. C.; Harnel, E.; Day, B. W.; Curran, D. P.; Org. Lett. 2005, 7, 2873.

9. Handa, M.; Scheidt, K. A.; Bossart, M.; Zheng, N.; Roush, W. R.; J. Org. Chem. 2008, 73, 1031.

10. O’Neil, G. W.; Fürstner, A.; Chem. Commun. 2008, 4294.

11. Shimp, H.; Micalizio, G. C.; Tetrahedron 2009, 65, 5908.

12. Cook, C.; Guinchard, X.; Liron, F.; Roulland, E.; Org. Lett. 2010, 12, 744 .

13. Trost, B. M.; Fettes, A.; Shireman, B. T.; J. Am. Chem. Soc. 2004, 126, 2660.

14. Silva, F.; Sawicki, M.; Gouverneur, V.; Org. Lett. 2006, 8, 5417.

15. List, B.; Lerner, R. A.; Barbas III, C. F.; J. Am. Chem. Soc. 2000, 122, 2395.
16. Tang, Z.; Jiang, F.; Yu, L.-T.; Cui, X.; Gong, L.-Z.; Mi, A.-Q.; Jiang, Y.-Z.; Wu, Y.-D.; J. Am. Chem. Soc. 2003, 125, 5262.

17. Tang, Z.; Jiang, F.; Cui, X.; Gong, L.-Z.; Mi, A.-Q.; Jiang, Y.-Z.; Wu, Y.-D.; Proc. Natl. Acad. Sci. U. S. A. 2004, 101, 5755.

18. Tang, Z.; Yang, Z.-H.; Chen, X.-H.; Cun, L.-F.; Mi, A.-Q.; Jiang, Y.-Z.; Gong, L.-Z.; J. Am. Chem. Soc. 2005, 127, 9285.

19. List, B.; Acc. Chem. Res. 2004, 37, 548.

20. Notz, W.; Tanaka, F.; Barbas III, C. F.; Acc. Chem. Res. 2004, 37,580 .

21. List, B.; Tetrahedron 2002, 58, 5573.

22. Pellissier, H.; Tetrahedron 2007, 63, 9267.

23. Guo, Q.; Bhanushali, M.; Zhao,C.-G.; Angew. Chem., Int. Ed. 2010, 49, 9460.

24. Allu, S.; Molleti, N.; Panem, R.; Singh, V. K.; Tetrahedron Lett. 2011, 52, 4080.

25. Doyle, A. G.; Jacobsen, E. N.; Chem. Rev. 2007, 107, 5713.

26. Paradowska, J.; Stodulski, M.; Mlynarski, J.; Angew. Chem., Int. Ed. 2009, 48, 4288.

27. For a review see: Zhou, F.; Liu, Y.-L.; and Zhou, J.; Adv. Synth. Catal. 2010, 352, 1381.

28. Chen, J.-R.; Liu, X.-P.; Zhu, X.-Y.; Li, L.; Qiao, Y.-F.; Zhang, J.-M.; Xiao, W.-J.; Tetrahedron 2007, 63, 10437.

29. Malkov, A. V.; Kabeshov, M. A.; Bella, M.; Kysilka, O.; Malyshev, D. A.; Pluhackova, K.; Kocovsky, P.; Org. Lett. 2007, 9, 5473 .

30. Nakamura, S.; Hara, N.; Nakashima, H.; Kubo, K.; Shibata, N.; Toru, T.; Chem. Eur. J. 2008, 14, 8079.

31. Itoh, T.; Ishikawa, H.; Hayashi, Y.; Org. Lett. 2009, 11, 3854.

32. Angelici, G.; Corrêa, R. J.; Garden, S. J.; Tomasini, C.; Tetrahedron Lett. 2009, 50, 814.

33. Raj, M.; Veerasamy, N.; Singh, V. K.; Tetrahedron Lett. 2010 , $51,2157$.

34. Chen, W.-B.; Du, X.-L.; Cun, L.-F.; Zhang, X.-M.; Yuan, W.-C.; Tetrahedron 2010, 66, 1441.

35. Hara, N.; Nakamura, S.; Shibata, N.; Toru, T.; Adv. Synth. Catal. 2010, 352, 1621.

36. Luppi, G.; Monari, M.; Corrêa, R. J.; Violante, F. D.; Pinto, A. C.; Kaptein, B.; Broxterman, Q. B.; Garden, S. J.; Tomasini, C.; Tetrahedron 2006, 62, 12017.

37. Corrêa, R. J.; Garden, S. J.; Angelici, G.; Tomasini, C.; Eur. J. Org. Chem. 2008, 4, 736.

38. Goehring, R. R.; Sachdeva, Y. P.; Pisipati, J. S.; Sleevi, M. C.; Wolfe, J. F.; J. Am. Chem. Soc. 1985, 107, 435.

39. Tokunaga, T.; Hume,W. E.; Nagamine, J.; Kawamura, T.; Taiji, M.; Nagata, R.; Bioorg. Med. Chem. Lett. 2005, 15, 1789.

Submitted: September 15, 2011 Published online: November 17, 2011 


\title{
Asymmetric Organocatalytic Synthesis of $\beta$-Hydroxyynones with a Quaternary Carbon Center under Aqueous Conditions
}

\author{
Guowei Kang, Jun Jiang, * Hongxin Liu and Huayue Wu* \\ College of Chemistry and Materials Engineering, Wenzhou University, Chashan University Town, \\ Wenzhou, Zhejiang Province 325035, People's Republic of China
}

\section{Experimental}

General

Nuclear magnetic resonance (NMR) spectra were recorded on a Bruker-500 $\mathrm{MHz}$ spectrometer. High-resolution mass spectrometry (HRMS, Micromass GCT-MS) spectra were recorded on a P-SIMS-Gly from Bruker Daltonics Inc. Infrared (IR) spectra were recorded on a Nicolet MX-1E FT-IR spectromter. High performance liquid chromatography (HPLC) analysis was performed on a Shimadzu LC-20AT series HPLC. Chiralpak AS, $\mathrm{AD}, \mathrm{OD}$ and IA were purchased from Daicel Chemical Industries, Ltd. Optical rotation data were acquired on a PolAAr 3005 automatic polarimeter. Solvents for the column chromatography were distilled before use.

Commercial reagents were used as received, unless otherwise stated. $\alpha$-Keto ester 21-2o were prepared as reported. ${ }^{1}$ Thiourea 3a was prepared according to the procedure of Vakulya et al. ${ }^{2}$ Catalyst $3 \mathbf{b}-3 \mathbf{e}$ were prepared according to the literature of Arjan et al. ${ }^{3}$ and Okino et al. ${ }^{4}$

General procedure for the asymmetric organocatalytic synthesis of $\beta$-hydroxyynones with quaternary carbon center under aqueous condition

Active ketone $(0.1 \mathrm{mmol})$ and catalyst $3 \mathrm{e}(10 \mathrm{~mol} \%)$ were added to the saturated $\mathrm{CaCl}_{2}$ solution $(0.5 \mathrm{~mL})$ in a tube and the mixture was stirred at corresponding temperature for $15 \mathrm{~min}$, then the ynone $(0.5 \mathrm{mmol})$ was added and the resulting mixture was stirred for 9-72 $\mathrm{h}$ at determined temperature (monitored by thin layer chromatography (TLC)). The reaction mixture was extracted by $\mathrm{CH}_{2} \mathrm{Cl}_{2}(3 \times 0.5 \mathrm{~mL})$ and the organic phase was purified through flash column chromatography on a silica gel (eluent: petroleum ether/ethyl acetate $=5 / 1-3 / 1$ ) to yield pure products.

*e-mail: junjiang@wzu.edu.cn; huayuewu@wzu.edu.cn
1-Butyl-3-hydroxy-3-(2-oxo-4-phenylbut-3-ynyl)indolin2-one (4a)

Yield: $90 \%$; $[\alpha]_{\mathrm{D}}{ }^{15}=+5.56^{\circ}\left(c=0.232, \mathrm{CHCl}_{3}\right.$, ee $=80 \%$ ), determined by HPLC (Daicel Chiralpak IA, hexane/isopropanol $=90 / 10$, flow rate $\left.1.0 \mathrm{~mL} \mathrm{~min}^{-1}\right) \mathrm{t}_{\mathrm{R}} / \mathrm{min}$ 17.34 (minor), 18.58 (major); IR (KBr) $v_{\max } / \mathrm{cm}^{-1} 2955$, 2927, 2193, 1698, 1667, 1614, 1362, 1064, 753, 689; ${ }^{1} \mathrm{H} \mathrm{NMR}\left(500 \mathrm{MHz}, \mathrm{CDCl}_{3}\right) \delta 0.94(\mathrm{t}, 3 \mathrm{H}, J 7.4 \mathrm{~Hz}), 1.44-$ $1.36(\mathrm{~m}, 2 \mathrm{H}), 1.71-1.65(\mathrm{~m}, 2 \mathrm{H}), 3.32$ (d, 1H, J 16.7 Hz), $3.51(\mathrm{~d}, 1 \mathrm{H}, J 16.7 \mathrm{~Hz}), 3.66-3.60(\mathrm{~m}, 1 \mathrm{H}), 3.76-3.71(\mathrm{~m}$, $1 \mathrm{H}), 4.10(\mathrm{~s}, 1 \mathrm{H}), 6.86(\mathrm{~m}, 1 \mathrm{H}), 7.07(\mathrm{~m}, 1 \mathrm{H}), 7.33-7.30$ (m, 1H), 7.38-7.35 (m, 2H), 7.47-7.44 (m, 2H), 7.53-7.51 $(\mathrm{m}, 2 \mathrm{H}) ;{ }^{13} \mathrm{C}$ NMR $\left(126 \mathrm{MHz}, \mathrm{CDCl}_{3}\right) \delta 13.70,20.10$, 29.15, 39.95, 51.72, 73.99, 87.84, 92.53, 108.91, 119.42, $122.93,124.24,128.59$, 129.22, 130.04, 131.03, 133.16, 143.11, 176.02, 183.76; HRMS (ESI) exact mass calcd. for $\left(\mathrm{C}_{22} \mathrm{H}_{21} \mathrm{NNaO}_{3}\right)^{+}$requires $\mathrm{m} / \mathrm{z} 370.1419$, found $\mathrm{m} / \mathrm{z}$ 370.1412 .

1-Butyl-4-chloro-3-hydroxy-3-(2-oxo-4-phenylbut-3-ynyl) indolin-2-one (4b)

Yield: $86 \% ;[\alpha]_{\mathrm{D}}{ }^{15}=+3.51^{\circ}\left(c=0.265, \mathrm{CHCl}_{3}\right.$, ee $=46 \%$ ), determined by HPLC (Daicel Chiralpak IA, hexane/isopropanol $=90 / 10$, flow rate $1.0 \mathrm{~mL} \mathrm{~min}^{-1}$ ) $\mathrm{t}_{\mathrm{R}} / \min 17.65$ (major), 20.67 (minor); IR (KBr) $v_{\max } / \mathrm{cm}^{-1}$ 2959, 2200, 1705, 1668, 1607, 1362, 1131, 1067, 774, 759, 689; ${ }^{1} \mathrm{H}$ NMR $\left(500 \mathrm{MHz}, \mathrm{CDCl}_{3}\right) \delta 0.95(\mathrm{t}, 3 \mathrm{H}$, $J 7.4 \mathrm{~Hz}), 1.45-1.37(\mathrm{~m}, 2 \mathrm{H}), 1.71-1.65(\mathrm{~m}, 2 \mathrm{H}), 3.58$ (s, 1H), 3.64-3.60(m, 2H), 3.77-3.72 (m, 1H), $4.02(\mathrm{~d}$, $1 \mathrm{H}, J 16.6 \mathrm{~Hz}), 6.77-6.76(\mathrm{~m}, 1 \mathrm{H}), 6.99-6.97(\mathrm{~m}, 1 \mathrm{H})$, 7.26-7.23 (m, 1H), 7.38-7.35 (m, 2H), 7.47-7.44 (m, 1H), 7.54-7.52 (m, 2H); ${ }^{13} \mathrm{C}$ NMR (126 MHz, $\left.\mathrm{CDCl}_{3}\right) \delta 13.70$, 20.09, 28.98, 40.24, 50.17, 74.39, 87.50, 92.01, 107.45, $119.50,123.79,125.15,128.58,130.96,131.21,131.50$, 133.16, 145.48, 175.57, 182.73; HRMS (ESI) exact mass calcd. for $\left(\mathrm{C}_{22} \mathrm{H}_{20} \mathrm{ClNNaO}_{3}\right)^{+}$requires $m / z$ 404.1029, found $\mathrm{m} / \mathrm{z} 404.1031$. 
4-Bromo-1-butyl-3-hydroxy-3-(2-oxo-4-phenylbut-3-ynyl) indolin-2-one (4c)

Yield: $97 \% ;[\alpha]_{\mathrm{D}}{ }^{15}=+1.96^{\circ}\left(c=0.271, \mathrm{CHCl}_{3}\right.$, ee $=54 \%)$, determined by HPLC (Daicel Chiralpak AS-H, hexane/isopropanol $=70 / 30$, flow rate $\left.1.0 \mathrm{~mL} \mathrm{~min}^{-1}\right) \mathrm{t}_{\mathrm{R}} / \mathrm{min}$ 15.18 (major), 27.97 (minor); IR (KBr) $v_{\max } / \mathrm{cm}^{-1} 2952,2198$, $1705,1666,1603,1362,1126,1067,760,688 ;{ }^{1} \mathrm{H}$ NMR $\left(500 \mathrm{MHz}, \mathrm{CDCl}_{3}\right) \delta 0.95(\mathrm{t}, 3 \mathrm{H}, J 7.0 \mathrm{~Hz}), 1.41-1.38(\mathrm{~m}$, $2 \mathrm{H}), 1.69-1.66(\mathrm{~m}, 2 \mathrm{H}), 3.46(\mathrm{~m}, 1 \mathrm{H}), 3.62-3.58(\mathrm{~m}, 2 \mathrm{H})$, 3.76-3.72 (m, 1H), 4.09 (d, 1H, J 16.6 Hz), 6.81-6.80 (m, $1 \mathrm{H}), 7.18-7.15(\mathrm{~m}, 2 \mathrm{H}), 7.38-7.35(\mathrm{~m}, 2 \mathrm{H}), 7.45-7.44(\mathrm{~m}$, $1 \mathrm{H})$, 7.54-7.52 (m, 2H); $\left.{ }^{13} \mathrm{C} \mathrm{NMR} \mathrm{(126} \mathrm{MHz,} \mathrm{CDCl}_{3}\right)$ $\delta$ 13.70, 20.10, 28.95, 40.16, 50.02, 74.99, 87.54, 92.00, $107.97,119.45,119.52,126.81,126.85,128.58,130.95$, 131.39, 133.16, 145.71, 175.52, 182.68; HRMS (ESI) exact mass calcd. for $\left(\mathrm{C}_{22} \mathrm{H}_{20} \mathrm{BrNNaO}_{3}\right)^{+}$requires $m / z$ 448.0524, found $\mathrm{m} / \mathrm{z} 448.0525$.

1-Butyl-3-hydroxy-5-methyl-3-(2-oxo-4-phenylbut-3-ynyl) indolin-2-one (4d)

Yield: $90 \% ;[\alpha]_{\mathrm{D}}{ }^{15}=+1.65^{\circ}\left(c=0.315, \mathrm{CHCl}_{3}\right.$, ee $=77 \%$ ), determined by HPLC (Daicel Chiralpak OD-H, hexane/isopropanol $=90 / 10$, flow rate $1.0 \mathrm{~mL} \mathrm{~min}^{-1}$ ) $\mathrm{t}_{\mathrm{R}} / \min 13.92$ (major), 16.34 (minor); IR (KBr) $v_{\max } / \mathrm{cm}^{-1}$ 2595, 2923, 2198, 1698, 1669, 1496, 1361, 1124, 809, 759, 689; ${ }^{1} \mathrm{H} \mathrm{NMR}\left(500 \mathrm{MHz}, \mathrm{CDCl}_{3}\right) \delta 0.94(\mathrm{t}, 3 \mathrm{H}, J 7.4 \mathrm{~Hz})$, 1.43-1.35 (m, 2H), 1.70-1.64 (m, 2H), 2.31 (s, 3H), 3.27 (d, $1 \mathrm{H}, J 16.7 \mathrm{~Hz}), 3.47$ (d, 1H, J 16.7 Hz), 3.65-3.59 $(\mathrm{m}, 1 \mathrm{H}), 3.74-3.68(\mathrm{~m}, 1 \mathrm{H}), 3.83(\mathrm{~s}, 1 \mathrm{H}), 6.75-6.74(\mathrm{~m}$, $1 \mathrm{H}), 7.12-7.10(\mathrm{~m}, 1 \mathrm{H}), 7.27(\mathrm{~s}, 1 \mathrm{H}), 7.39-7.36(\mathrm{~m}, 2 \mathrm{H})$, 7.48-7.45 (m, 1H), 7.54-7.52 (m, 2H); ${ }^{13} \mathrm{C} \mathrm{NMR}(126 \mathrm{MHz}$, $\left.\mathrm{CDCl}_{3}\right) \delta 13.71,20.10,21.00,29.18,39.96,51.70,74.13$, $87.88,92.55,108.69,119.44,125.06,128.60,129.20$, 130.26, 131.04, 132.56, 133.16, 140.64, 175.84, 183.94; HRMS (ESI) exact mass calcd. for $\left(\mathrm{C}_{23} \mathrm{H}_{23} \mathrm{NNaO}_{3}\right)^{+}$requires $m / z$ 384.1576, found $m / z$ 384.1573.

1-Butyl-5-fluoro-3-hydroxy-3-(2-oxo-4-phenylbut-3-ynyl) indolin-2-one (4e)

Yield: $98 \% ;[\alpha]_{\mathrm{D}}{ }^{15}=+1.70^{\circ}\left(c=0.259, \mathrm{CHCl}_{3}\right.$, ee $=70 \%$ ), determined by HPLC (Daicel Chiralpak AS-H, hexane/isopropanol $=80 / 20$, flow rate $1.0 \mathrm{~mL} \mathrm{~min}^{-1}$ ) $\mathrm{t}_{\mathrm{R}} / \min 15.24$ (major), 1.53 (minor); IR (KBr) $\mathrm{v}_{\max } / \mathrm{cm}^{-1}$ 2959, 2200, 1704, 1669, 1489, 1362, 1065, 760, 689; ${ }^{1} \mathrm{H}$ NMR (500 MHz, $\left.\mathrm{CDCl}_{3}\right) \delta 0.94(\mathrm{t}, 3 \mathrm{H}, J 7.4 \mathrm{~Hz}), 1.43-$ 1.35(m, 2H), 1.69-1.63 (m, 2H), $3.33(\mathrm{~d}, 1 \mathrm{H}, J 16.9 \mathrm{~Hz})$, 3.51 (d, 1H, J 16.9 Hz), 3.64-3.58 (m, 1H), 3.75-3.70 (m, $1 \mathrm{H}), 4.25-4.20(\mathrm{~m}, 1 \mathrm{H}), 6.79-6.77(\mathrm{~m}, 1 \mathrm{H}), 7.03-6.99(\mathrm{~m}$,
1H), 7.22-7.20 (m, 1H), 7.38-7.35 (m, 2H), 7.47-7.44 (m, 1H), 7.54-7.52 (m, 2H); ${ }^{13} \mathrm{C}$ NMR (126 MHz, $\left.\mathrm{CDCl}_{3}\right)$ $\delta$ 13.69, 20.09, 29.06, 40.12, 51.66, 74.04, 87.71, 92.90, $109.49,109.55,112.51,112.71,116.12,116.31,119.29$, $128.64,130.76,130.82,131.15,133.19,139.01,158.31$, 160.23, 175.79, 183.53; HRMS (ESI) exact mass calcd. for $\left(\mathrm{C}_{22} \mathrm{H}_{20} \mathrm{FNNaO}_{3}\right)^{+}$requires $m / z 388.1325$, found $m / z 388.1321$.

1-Butyl-5-chloro-3-hydroxy-3-(2-oxo-4-phenylbut-3-ynyl) indolin-2-one (4f)

Yield: $90 \% ;[\alpha]_{\mathrm{D}}{ }^{15}=+1.08^{\circ}\left(c=0.358, \mathrm{CHCl}_{3}\right.$, ee $=66 \%$ ), determined by HPLC (Daicel Chiralpak AS-H, hexane/isopropanol $=80 / 20$, flow rate $\left.1.0 \mathrm{~mL} \mathrm{~min}^{-1}\right) \mathrm{t}_{\mathrm{R}} / \mathrm{min}$ 14.68 (major), 18.29 (minor); IR (KBr) $v_{\max } / \mathrm{cm}^{-1} 2955,2199$, 1705, 1668, 1487, 1362, 1111, 1063, 758, 688; ${ }^{1} \mathrm{H}$ NMR $\left(500 \mathrm{MHz}, \mathrm{CDCl}_{3}\right) \delta 0.94(\mathrm{t}, 3 \mathrm{H}, J 7.4 \mathrm{~Hz}), 1.43-1.35(\mathrm{~m}$, 2H), 1.69-1.63 (m, 2H), 3.33 (d, 1H, J 17.0 Hz), $3.52(\mathrm{~d}, 1 \mathrm{H}$, $J$ 17.0 Hz), 3.64-3.58 (m, 1H), 3.75-3.69 (m, 1H), 4.04 (s, $1 \mathrm{H}), 6.79-6.78(\mathrm{~m}, 1 \mathrm{H}), 7.30-7.28(\mathrm{~m}, 1 \mathrm{H}), 7.39-7.36(\mathrm{~m}$, 2H), 7.44-7.43 (m, 1H), 7.48-7.45 (m, 1H), 7.55-7.53 (m, 2H); ${ }^{13} \mathrm{C} \mathrm{NMR}\left(126 \mathrm{MHz}, \mathrm{CDCl}_{3}\right) \delta 13.70,20.09,29.07,40.12$, 51.57, 73.86, 87.67, 93.03, 109.91, 119.27, 124.89, 128.35, $128.66,129.94,130.87,131.18,133.21,141.75,175.52$, 183.54; HRMS (ESI) exact mass calcd. for $\left(\mathrm{C}_{22} \mathrm{H}_{20} \mathrm{ClNNaO}_{3}\right)^{+}$ requires $m / z$ 404.1029, found $m / z$ 404.1024.

5-Bromo-1-butyl-3-hydroxy-3-(2-oxo-4-phenylbut-3-ynyl) indolin-2-one (4g)

Yield: $90 \% ;[\alpha]_{\mathrm{D}}{ }^{15}=-0.71^{\circ}\left(c=0.225, \mathrm{CHCl}_{3}\right.$, ee $=66 \%$ ), determined by HPLC (Daicel Chiralpak AS-

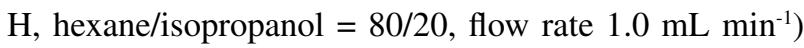
$\mathrm{t}_{\mathrm{R}} / \min 15.29$ (major), 18.28 (minor); IR (KBr) $\mathrm{v}_{\max } / \mathrm{cm}^{-1}$ 2952, 2199, 1705, 1668, 1361, 1222, 1123, 1059, 758, 688; ${ }^{1} \mathrm{H}$ NMR $\left(500 \mathrm{MHz}, \mathrm{CDCl}_{3}\right) \delta 0.93(\mathrm{t}, 3 \mathrm{H}, J 7.4 \mathrm{~Hz}), 1.42-$ 1.34 (m, 2H), 1.68-1.62 (m, 2H), 3.36 (d, 1H, J 17.0 Hz), $3.52(\mathrm{~d}, 1 \mathrm{H}, J 17.0 \mathrm{~Hz}), 3.62-3.56(\mathrm{~m}, 1 \mathrm{H}), 3.74-3.69(\mathrm{~m}$, $1 \mathrm{H}), 4.19(\mathrm{~s}, 1 \mathrm{H}), 6.74-6.72(\mathrm{~m}, 1 \mathrm{H}), 7.38-7.35$ (m, 2H), 7.47-7.42 (m, 2H), 7.56-7.52 (m, 3H); ${ }^{13} \mathrm{C} \mathrm{NMR} \mathrm{(126} \mathrm{MHz,}$ $\left.\mathrm{CDCl}_{3}\right) \delta 13.69,20.08,29.04,40.09,51.65,73.78,87.65$, 92.96, 110.40, 115.57, 119.27, 127.57, 128.64, 131.15, 131.24, 132.84, 133.20, 142.27, 175.52,183.41; HRMS (ESI) exact mass calcd. for $\left(\mathrm{C}_{22} \mathrm{H}_{20} \mathrm{BrNNaO}_{3}\right)^{+}$requires $\mathrm{m} / \mathrm{z}$ 448.0524 , found $\mathrm{m} / \mathrm{z} 448.0521$.

1-Butyl-3-hydroxy-5-nitro-3-(2-oxo-4-phenylbut-3-ynyl) indolin-2-one (4h)

Yield: $89 \% ;[\alpha]_{\mathrm{D}}{ }^{15}=-0.64^{\circ}\left(c=0.326, \mathrm{CHCl}_{3}\right.$, ee $=57 \%$ ), determined by HPLC (Daicel Chiralpak OD-H, 
hexane/isopropanol $=90 / 10$, flow rate $1.0 \mathrm{~mL} \mathrm{~min}^{-1}$ ) $\mathrm{t}_{\mathrm{R}} / \min 28.49$ (major), 36.37 (minor); IR (KBr) $v_{\max } / \mathrm{cm}^{-1}$ 2955, 2930, 2196, 1728, 1704, 1667, 1614, 1519, 1330, $1068,756,689 ;{ }^{1} \mathrm{H}$ NMR $\left(500 \mathrm{MHz}, \mathrm{CDCl}_{3}\right) \delta 0.96(\mathrm{t}, 3 \mathrm{H}$, $J 7.3 \mathrm{~Hz}), 1.46-1.38(\mathrm{~m}, 2 \mathrm{H}), 1.74-1.67(\mathrm{~m}, 2 \mathrm{H}), 3.48(\mathrm{~d}$, $1 \mathrm{H}, J 17.5 \mathrm{~Hz}), 3.63(\mathrm{~d}, 1 \mathrm{H}, J 17.5 \mathrm{~Hz}), 3.71-3.67(\mathrm{~m}, 1 \mathrm{H})$, 3.85-3.79 (m, 1H), 4.07-3.98 (m, 1H), 6.96-6.94 (m, 1H), 7.38-7.35 (m, 2H), 7.48-7.45 (m, 1H), 7.54-7.52 (m, 2H), 8.30-8.29 (m, 2H); ${ }^{13} \mathrm{C}$ NMR (126 MHz, $\left.\mathrm{CDCl}_{3}\right) \delta 13.66$, 20.08, 29.07, 40.51, 51.74, 73.08, 87.37, 93.43, 108.55, 119.09, 120.07, 127.17, 128.70, 130.05, 131.31, 133.21, 143.52, 149.24, 176.46,182.87; HRMS (ESI) exact mass calcd. for $\left(\mathrm{C}_{22} \mathrm{H}_{20} \mathrm{~N}_{2} \mathrm{NaO}_{5}\right)^{+}$requires $\mathrm{m} / z 415.1270$, found $\mathrm{m} / \mathrm{z} 415.1264$.

1-Butyl-3-hydroxy-5-methoxy-3-(2-oxo-4-phenylbut-3-ynyl) indolin-2-one (4i)

Yield: $94 \% ;[\alpha]_{D}{ }^{15}=+1.37^{\circ}\left(c=0.277, \mathrm{CHCl}_{3}\right.$, ee $=78 \%$ ), determined by HPLC (Daicel Chiralpak OD$\mathrm{H}$, hexane/isopropanol $=80 / 20$, flow rate $\left.1.0 \mathrm{~mL} \mathrm{~min}^{-1}\right)$ $\mathrm{t}_{\mathrm{R}} /$ min 10.62 (major), 14.88 (minor); IR (KBr) $v_{\max } / \mathrm{cm}^{-1}$ 2933, 2193, 1694, 1667, 1489, 1352, 1284, 1036, 758, 689; ${ }^{1} \mathrm{H}$ NMR (500 MHz, $\left.\mathrm{CDCl}_{3}\right) \delta 0.93(\mathrm{t}, 3 \mathrm{H}, J 7.4 \mathrm{~Hz}$ ), 1.43-1.35 (m, 2H), 1.69-1.63 (m, 2H), $3.29(\mathrm{~d}, 1 \mathrm{H}$, $J 16.7 \mathrm{~Hz}), 3.49(\mathrm{~d}, 1 \mathrm{H}, J 16.7 \mathrm{~Hz}), 3.64-3.57(\mathrm{~m}, 1 \mathrm{H})$, 3.74-3.68 (m, 1H), $3.77(\mathrm{~s}, 3 \mathrm{H}), 4.09(\mathrm{~s}, 1 \mathrm{H}), 6.77-6.75$ (m, 1H), 6.85-6.82 (m, 1H), 7.09-7.08 (m, 1H), 7.38-7.35 (m, 2H), 7.47-7.44 (m, 1H), 7.54-7.52 (m, 2H); ${ }^{13} \mathrm{C} \mathrm{NMR}$ $(126 \mathrm{MHz}, \mathrm{CDCl} 3) \delta 13.73,20.12,29.19,40.03,51.68$, 55.82, 74.39, 87.88, 92.67, 109.43, 111.49, 114.64, 119.42, 128.62, 130.39, 131.07, 133.18, 136.34, 156.18, 175.60, 183.89; HRMS (ESI) exact mass calcd. for $\left(\mathrm{C}_{23} \mathrm{H}_{23} \mathrm{NNaO}_{4}\right)^{+}$ requires $m / z$ 400.1525, found $m / z 400.1521$.

6-Bromo-1-butyl-3-hydroxy-3-(2-oxo-4-phenylbut-3-ynyl) indolin-2-one (4j)

Yield: $97 \% ;[\alpha]_{\mathrm{D}}{ }^{15}=+4.14^{\circ}\left(c=0.406, \mathrm{CHCl}_{3}\right.$, ee $=70 \%$ ), determined by HPLC (Daicel Chiralpak IA, hexane/isopropanol $=90 / 10$, flow rate $1.0 \mathrm{~mL} \mathrm{~min}^{-1}$ ) $\mathrm{t}_{\mathrm{R}} /$ min 14.83 (minor), 16.84 (major); IR (KBr) $v_{\max } / \mathrm{cm}^{-1}$ 2955, 2930, 2198, 1708, 1668, 1606, 1488, 1363, 1061, 758, 688; ${ }^{1} \mathrm{H}$ NMR $\left(500 \mathrm{MHz}, \mathrm{CDCl}_{3}\right) \delta 0.95(\mathrm{t}, 3 \mathrm{H}$, $J 7.4 \mathrm{~Hz}), 1.43-1.36(\mathrm{~m}, 2 \mathrm{H}), 1.69-1.63(\mathrm{~m}, 2 \mathrm{H}), 3.31(\mathrm{~d}$, $1 \mathrm{H}, J 16.9 \mathrm{~Hz}), 3.50$ (d, $1 \mathrm{H}, J 16.9 \mathrm{~Hz}), 3.62-3.56(\mathrm{~m}, 1 \mathrm{H})$, 3.73-3.67 (m, 1H), 4.10-3.98 (m, 1H), 7.00-6.99 (m, 1H), 7.20-7.19 (m, 1H), 7.30-7.29 (m, 1H), 7.39-7.36 (m, 2H), 7.48-7.45 (m, 1H), 7.53-7.51 (m, 2H); ${ }^{13} \mathrm{C} \mathrm{NMR}(126 \mathrm{MHz}$, $\left.\mathrm{CDCl}_{3}\right) \delta 13.69,20.10,29.07,40.15,51.53,73.62,87.72$, $93.00,112.41,119.29,123.88,125.59,125.74,128.15$,
128.67, 131.19, 133.21, 144.57, 175.77,183.60; HRMS (ESI) exact mass calcd. for $\left(\mathrm{C}_{22} \mathrm{H}_{20} \mathrm{BrNNaO}_{3}\right)^{+}$requires $\mathrm{m} / \mathrm{z}$ 448.0524, found $\mathrm{m} / \mathrm{z} 448.0523$.

1-Butyl-7-fluoro-3-hydroxy-3-(2-oxo-4-phenylbut-3-ynyl) indolin-2-one (4k)

Yield: $95 \% ;[\alpha]_{\mathrm{D}}^{15}=+3.61^{\circ}\left(c=0.338, \mathrm{CHCl}_{3}\right.$, ee $=65 \%$ ), determined by HPLC (Daicel Chiralpak IA, hexane/isopropanol $=85 / 15$, flow rate $\left.1.0 \mathrm{~mL} \mathrm{~min}^{-1}\right) \mathrm{t}_{\mathrm{R}} / \mathrm{min}$ 9.02 (minor), 10.33 (major); IR (KBr) $v_{\max } / \mathrm{cm}^{-1} 2959,2200$, 1705, 1669, 1362, 1126,1058, 759, 732, 689; ${ }^{1} \mathrm{H}$ NMR $\left(500 \mathrm{MHz}, \mathrm{CDCl}_{3}\right) \delta 0.94(\mathrm{t}, 3 \mathrm{H}, J 7.4 \mathrm{~Hz}), 1.44-1.36(\mathrm{~m}$, $2 \mathrm{H}), 1.72-1.67(\mathrm{~m}, 2 \mathrm{H}), 3.35(\mathrm{~d}, 1 \mathrm{H}, J 16.9 \mathrm{~Hz}), 3.51(\mathrm{~d}$, 1H, J 16.9 Hz), 3.89-3.77 (m, 2H), 4.14 (s, 1H), 7.08-6.99 (m, 2H), 7.24-7.22 (m, 1H), 7.39-7.36 (m, 2H), 7.48-7.44 (m, 1H), 7.54-7.52 (m, 2H); $\left.{ }^{13} \mathrm{C} \mathrm{NMR} \mathrm{(126} \mathrm{MHz,} \mathrm{CDCl}_{3}\right)$ $\delta$ 13.70, 19.88, 30.66, 30.68, 42.01, 42.05, 51.75, 74.01, 74.03, 87.74, 92.86, 118.21, 118.37, 119.34, 119.99, $120.02,123.73,123.79,128.65,129.77,129.84,131.15$, $132.15,132.17,133.20,146.55,148.50,175.66,183.56$; HRMS (ESI) exact mass calcd. for $\left(\mathrm{C}_{22} \mathrm{H}_{20} \mathrm{FNNaO}_{3}\right)^{+}$ requires $\mathrm{m} / \mathrm{z} 388.1325$, found $\mathrm{m} / \mathrm{z} 388.1318$.

1-Butyl-3-hydroxy-3-(2-oxo-4-p-tolylbut-3-ynyl)indolin2-one (4ba)

Yield: $53 \% ;[\alpha]_{D}{ }^{15}=+12.4^{\circ}\left(c=0.062, \mathrm{CHCl}_{3}\right.$, ee $=78 \%$ ), determined by HPLC (Daicel Chiralpak AS-H, hexane/isopropanol $=80 / 20$, flow rate $1.0 \mathrm{~mL} \mathrm{m^{-1 }}$ ) $\mathrm{t}_{\mathrm{R}} / \mathrm{min} 13.03$ (major), 18.40 (minor); IR (KBr) $v_{\max } / \mathrm{cm}^{-1}$ 2955, 2199, 1704, 1669, 1362, 1062, 817, 753; ${ }^{1} \mathrm{H}$ NMR $\left(500 \mathrm{MHz}, \mathrm{CDCl}_{3}\right) \delta 0.94(\mathrm{t}, 3 \mathrm{H}, J 7.4 \mathrm{~Hz}), 1.44-1.36$ $(\mathrm{m}, 2 \mathrm{H}), 1.70-1.65(\mathrm{~m}, 2 \mathrm{H}), 2.38(\mathrm{~s}, 3 \mathrm{H}), 3.28(\mathrm{~d}, 1 \mathrm{H}$, $J 16.7 \mathrm{~Hz}), 3.49(\mathrm{~d}, 1 \mathrm{H}, J 16.7 \mathrm{~Hz}), 3.68-3.60(\mathrm{~m}, 1 \mathrm{H})$, 3.76-3.70 (m, 1H), 4.05-4.01 (m, 1H), 6.86-6.84 (m, 1H), 7.07-7.04 (m, 1H), 7.18-7.16 (m, 2H), 7.33-7.30 (m, 1H), 7.45-7.42 (m, 3H); ${ }^{13} \mathrm{C}$ NMR (126 MHz, $\left.\mathrm{CDCl}_{3}\right) \delta 13.72$, 20.13, 21.75, 29.20, 39.95, 51.47, 74.10, 87.85, 93.53, 108.90, 116.29, 122.92, 124.31, 129.30, 129.44, 130.05, 133.26, 141.94, 143.10, 175.81, 184.08; HRMS (ESI) exact mass calcd. for $\left(\mathrm{C}_{23} \mathrm{H}_{23} \mathrm{NNaO}_{3}\right)^{+}$requires $\mathrm{m} / \mathrm{z} 384.1576$, found $m / z 384.1574$.

\section{1-Butyl-3-hydroxy-3-(2-oxobut-3-ynyl)indolin-2-one (4ca)}

Yield: 70\%; IR (KBr) $v_{\max } / \mathrm{cm}^{-1} 3234,2952,2923$, 2087, 1682, 1614, 1338, 1125, 1099, 752; ${ }^{1} \mathrm{H}$ NMR $\left(500 \mathrm{MHz}, \mathrm{CDCl}_{3}\right) \delta 0.96(\mathrm{t}, 3 \mathrm{H}, \mathrm{J} 7.4 \mathrm{~Hz}), 1.44-1.40(\mathrm{~m}$, $2 \mathrm{H}), 1.69-1.65(\mathrm{~m}, 2 \mathrm{H}), 3.24(\mathrm{~d}, 1 \mathrm{H}, J 17.1 \mathrm{~Hz}), 3.27(\mathrm{~s}$, $1 \mathrm{H}), 3.43(\mathrm{~d}, 1 \mathrm{H}, J 17.1 \mathrm{~Hz}), 3.66-3.60(\mathrm{~m}, 1 \mathrm{H}), 3.76-3.70$ 
(m, 1H), $3.76(\mathrm{~s}, 1 \mathrm{H}), 6.86-6.85(\mathrm{~m}, 1 \mathrm{H}), 7.08-7.05(\mathrm{~m}$, $1 \mathrm{H})$, 7.34-7.31 (m, 1H), 7.40-7.38 (m, 1H); ${ }^{13} \mathrm{C}$ NMR $\left(126 \mathrm{MHz}, \mathrm{CDCl}_{3}\right) \delta 13.72,20.13,29.17,39.99,51.67$, 73.69, 79.99, 81.20, 108.98, 122.96, 124.13, 128.91, 130.21, 143.21, 175.77,183.20; HRMS (ESI) exact mass calcd. for $\left(\mathrm{C}_{16} \mathrm{H}_{17} \mathrm{NNaO}_{3}\right)^{+}$requires $\mathrm{m} / z$ 294.1106, found $\mathrm{m} / \mathrm{z}, 294.1102$.

\section{Benzyl 2-hydroxy-4-oxo-2,6-diphenylhex-5-ynoate (4I)}

Yield: $42 \% ;[\alpha]_{\mathrm{D}}^{15}=+2.65^{\circ}\left(c=0.066, \mathrm{CHCl}_{3}\right.$, ee $=47 \%$ ), determined by HPLC (Daicel Chiralpak AD-H, hexane/isopropanol $=80 / 20$, flow rate $\left.1.0 \mathrm{~mL} \mathrm{~min}^{-1}\right)$ $\mathrm{t}_{\mathrm{R}} / \mathrm{min} 24.39$ (major), 30.26 (minor); IR (KBr) $v_{\max } / \mathrm{cm}^{-1}$ 2936, 2196, 1732, 1668, 1204, 1178, 1086, 757, 695; ${ }^{1} \mathrm{H}$ NMR (500 MHz, $\left.\mathrm{CDCl}_{3}\right) \delta 3.35(\mathrm{~d}, 1 \mathrm{H}, J 17.8 \mathrm{~Hz})$, $3.80(\mathrm{~d}, 1 \mathrm{H}, J 17.8 \mathrm{~Hz}), 4.24(\mathrm{~s}, 1 \mathrm{H}), 5.23-5.17(\mathrm{~m}, 2 \mathrm{H})$, 7.26-7.24 (m, 2H), 7.32-7.29 (m, 4H), 7.39-7.33 (m, 4H), 7.48-7.44 (m, 1H), 7.55-7.53(m, 2H),7.60-7.57(m, 2H); ${ }^{13} \mathrm{C}$ NMR $\left(126 \mathrm{MHz}, \mathrm{CDCl}_{3}\right) \delta 54.72,68.14,76.07,87.61$, $92.49,119.52,125.10,128.20,128.39,128.49,128.52$, $128.63,131.01,133.13,134.96,140.02,173.46,184.73$; HRMS (ESI) exact mass calcd. for $\left(\mathrm{C}_{25} \mathrm{H}_{20} \mathrm{NaO}_{4}\right)^{+}$requires $\mathrm{m} / \mathrm{z}$ 407.1259, found $\mathrm{m} / \mathrm{z}$ 407.1258.

Isopropyl 2-hydroxy-4-oxo-2,6-diphenylhex-5-ynoate (4m)

Yield: $36 \% ;[\alpha]_{\mathrm{D}}^{15}=+2.69^{\circ}\left(c=0.078, \mathrm{CHCl}_{3}\right.$, ee $=63 \%)$, determined by HPLC (Daicel Chiralpak AD-H, hexane/isopropanol $=90 / 10$, flow rate $\left.1.0 \mathrm{~mL} \mathrm{~min}^{-1}\right)$ $\mathrm{t}_{\mathrm{R}} / \min 18.79$ (major), 28.04 (minor); IR (KBr) $v_{\max } / \mathrm{cm}^{-1}$ 2923, 2190, 1722, 1670, 1263, 1219, 1104, 1085, 758, 689; ${ }^{1} \mathrm{H}$ NMR (500 MHz, $\left.\mathrm{CDCl}_{3}\right) \delta 1.27-1.24(\mathrm{~m}, 6 \mathrm{H}), 3.34(\mathrm{~d}$, $1 \mathrm{H}, J 17.8 \mathrm{~Hz}), 3.76$ (d, 1H, J 17.8 Hz), 4.19 (s, 1H), 5.10 (hept, $1 \mathrm{H}, J 6.3 \mathrm{~Hz}), 7.33-7.29(\mathrm{~m}, 1 \mathrm{H}), 7.40-7.35(\mathrm{~m}, 4 \mathrm{H})$, 7.48-7.44 (m, 1H), 7.57-7.54 (m, 2H), 7.64-7.61 (m, 2H); ${ }^{13} \mathrm{C} \mathrm{NMR}\left(126 \mathrm{MHz}, \mathrm{CDCl}_{3}\right) \delta 21.49,54.88,70.50,75.82$, 87.66, 92.07, 119.61, 125.05, 128.07, 128.41, 128.63, 130.95, 133.12, 140.48, 173.05, 184.48; HRMS (ESI) exact mass calcd. for $\left(\mathrm{C}_{21} \mathrm{H}_{20} \mathrm{NaO}_{4}\right)^{+}$requires $m / z 359.1259$, found $\mathrm{m} / \mathrm{z} 359.1256$.
Isobutyl 2-hydroxy-4-oxo-2,6-diphenylhex-5-ynoate (4n)

Yield: $34 \% ;[\alpha]_{\mathrm{D}}{ }^{15}=+3.13^{\circ}\left(c=0.083, \mathrm{CHCl}_{3}\right.$, ee $=62 \%)$, determined by HPLC (Daicel Chiralpak AD-H, hexane/isopropanol $=80 / 20$, flow rate $1.0 \mathrm{~mL} \mathrm{~min}^{-1}$ ) $\mathrm{t}_{\mathrm{R}} /$ min 12.56 (major), 15.99 (minor); IR (KBr) $v_{\max } / \mathrm{cm}^{-1}$ 2959, 2190, 1723, 1668, 1278, 1205, 1125, 1086, 758, 689; ${ }^{1} \mathrm{H}$ NMR $\left(500 \mathrm{MHz}, \mathrm{CDCl}_{3}\right) \delta 0.89(\mathrm{~d}, 6 \mathrm{H}, J 6.7 \mathrm{~Hz})$, 2.01-1.92 (m, 1H), $3.37(\mathrm{~d}, 1 \mathrm{H}, J 17.8 \mathrm{~Hz}), 3.80(\mathrm{~d}, 1 \mathrm{H}$, $J 17.8 \mathrm{~Hz}), 3.98$ (d, 2H, J 6.6 Hz), 4.24 (s, 1H), 7.33-7.30 $(\mathrm{m}, 1 \mathrm{H}), 7.40-7.36(\mathrm{~m}, 4 \mathrm{H}), 7.48-7.44(\mathrm{~m}, 1 \mathrm{H}), 7.57-7.55$ $(\mathrm{m}, 2 \mathrm{H}), 7.64-7.62(\mathrm{~m}, 2 \mathrm{H}) ;{ }^{13} \mathrm{C} \mathrm{NMR}\left(126 \mathrm{MHz}, \mathrm{CDCl}_{3}\right)$ $\delta$ 18.92, 18.96, 27.61, 54.71, 72.50, 75.94, 87.61, 92.28, 119.56, 125.10, 128.15, 128.45, 128.63, 130.98, 133.13, 140.35, 173.68,184.61; HRMS (ESI) exact mass calcd. for $\left(\mathrm{C}_{22} \mathrm{H}_{22} \mathrm{NaO}_{4}\right)^{+}$requires $m / z 373.1416$, found $m / z 373.1411$.

Ethyl 2-hydroxy-4-oxo-2,6-diphenylhex-5-ynoate (40)

Yield: $44 \% ;[\alpha]_{\mathrm{D}}{ }^{15}=+4.43^{\circ}\left(c=0.07, \mathrm{CHCl}_{3}\right.$, ee $=60 \%$ ), determined by HPLC (Daicel Chiralpak IA, hexane/isopropanol $=90 / 10$, flow rate $\left.1.0 \mathrm{~mL} \mathrm{~min}^{-1}\right)$ $\mathrm{t}_{\mathrm{R}} / \min 14.92$ (major), 16.93 (minor); IR ( $\left.\mathrm{KBr}\right) v_{\max } / \mathrm{cm}^{-1}$ 2962, 2923, 2200, 1731, 1669, 1363, 1261, 1221, 1085, 759, 689; ${ }^{1} \mathrm{H}$ NMR $\left(500 \mathrm{MHz}, \mathrm{CDCl}_{3}\right) \delta 1.28(\mathrm{t}, 3 \mathrm{H}$, $J 7.1 \mathrm{~Hz}), 3.34(\mathrm{~d}, 1 \mathrm{H}, J 17.8 \mathrm{~Hz}), 3.79(\mathrm{~d}, 1 \mathrm{H}, J 17.8 \mathrm{~Hz})$, $4.22(\mathrm{~s}, 1 \mathrm{H}), 4.27(\mathrm{q}, 2 \mathrm{H}, J 7.1 \mathrm{~Hz}), 7.34-7.31(\mathrm{~m}, 1 \mathrm{H})$, 7.40-7.37 (m, 4H), 7.48-7.45 (m, 1H), 7.57-7.55 (m, 2H), 7.63-7.62 (m, 2H); $\left.{ }^{13} \mathrm{C} \mathrm{NMR} \mathrm{(126} \mathrm{MHz,} \mathrm{CDCl}_{3}\right) \delta 13.96$, 54.90, 62.54, 75.94, 87.62, 92.29, 119.57, 125.05, 128.15, $128.48,128.64,130.99,133.14,140.31,173.57,184.69$; HRMS (ESI) exact mass calcd. for $\left(\mathrm{C}_{20} \mathrm{H}_{18} \mathrm{NaO}_{4}\right)^{+}$requires $\mathrm{m} / \mathrm{z}$ 345.1103, found $\mathrm{m} / \mathrm{z} 345.1107$.

\section{References}

1. Hu, S.; Neckers, D. C.; J. Org. Chem. 1996, 61, 6407; Di Mauro, E. F.; Kozlowshi, M. C.; J. Am. Chem. Soc. 2002, 124, 12668.

2. Vakulya, B.; Varga, S.; Csampai, A.; Soos, T.; Org. Lett. 2005 , 7, 1967.

3. Arjan, H.; Boyd, E.; Coumbarides, G. S.; Eames, J.; Jones, R. V.H.; Stensonc, R. A.; Suggate, M. J.; Tetrahedron Lett. 2005, 46, 1921.

4. Okino, T.; Hoashi, Y.; Takemoto, Y.; J. Am. Chem. Soc. 2003, $125,12672$. 

k.01001060!

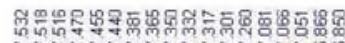
年

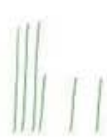

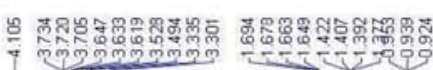

:
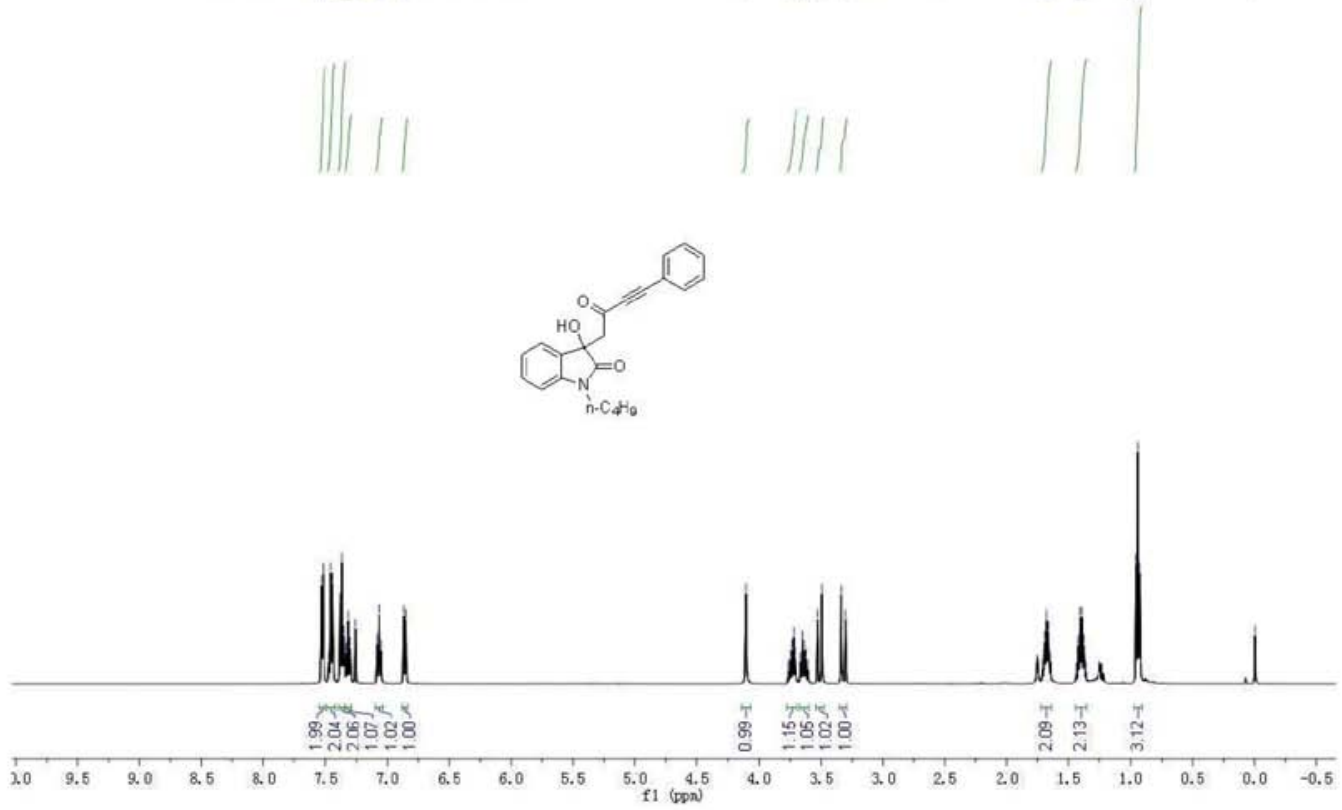

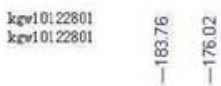

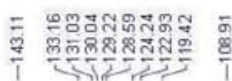

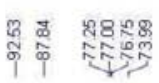

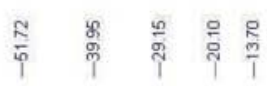

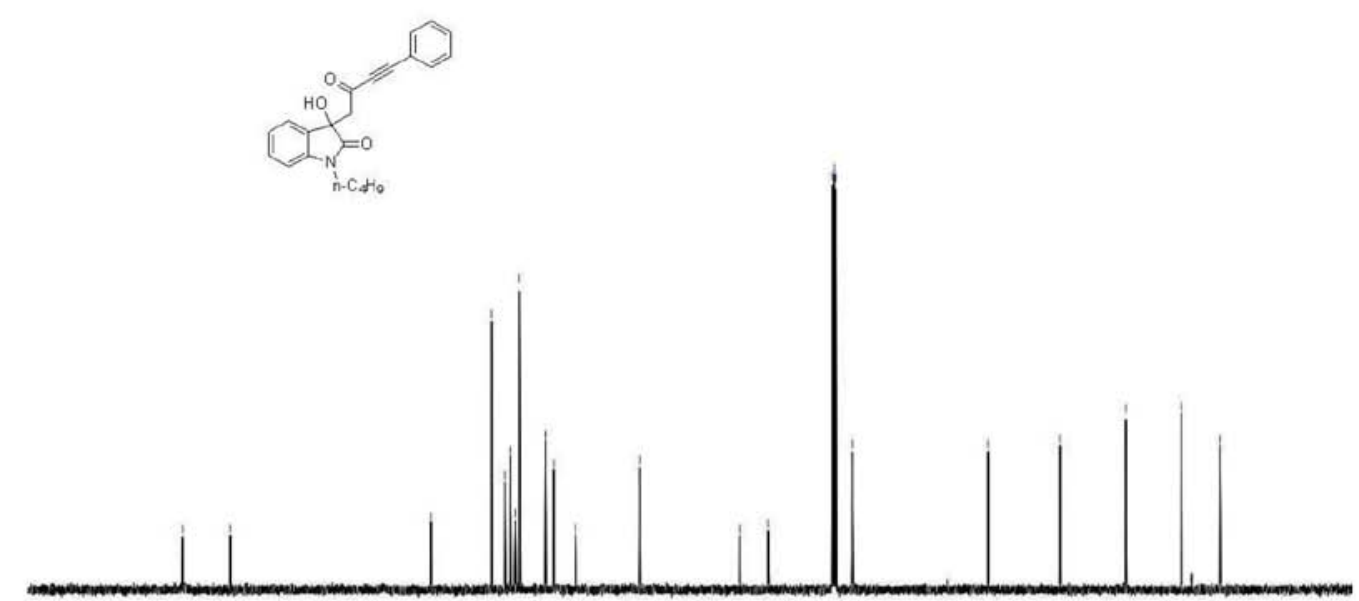
$110 \stackrel{100}{100}$

Figure S1. ${ }^{1} \mathrm{H}$ NMR spectrum $\left(500 \mathrm{MHz}, \mathrm{CDCl}_{3}\right)$ and ${ }^{13} \mathrm{C} \mathrm{NMR}$ spectrum $\left(126 \mathrm{MHz}, \mathrm{CDCl}_{3}\right)$ of compound $4 \mathbf{a}$. 

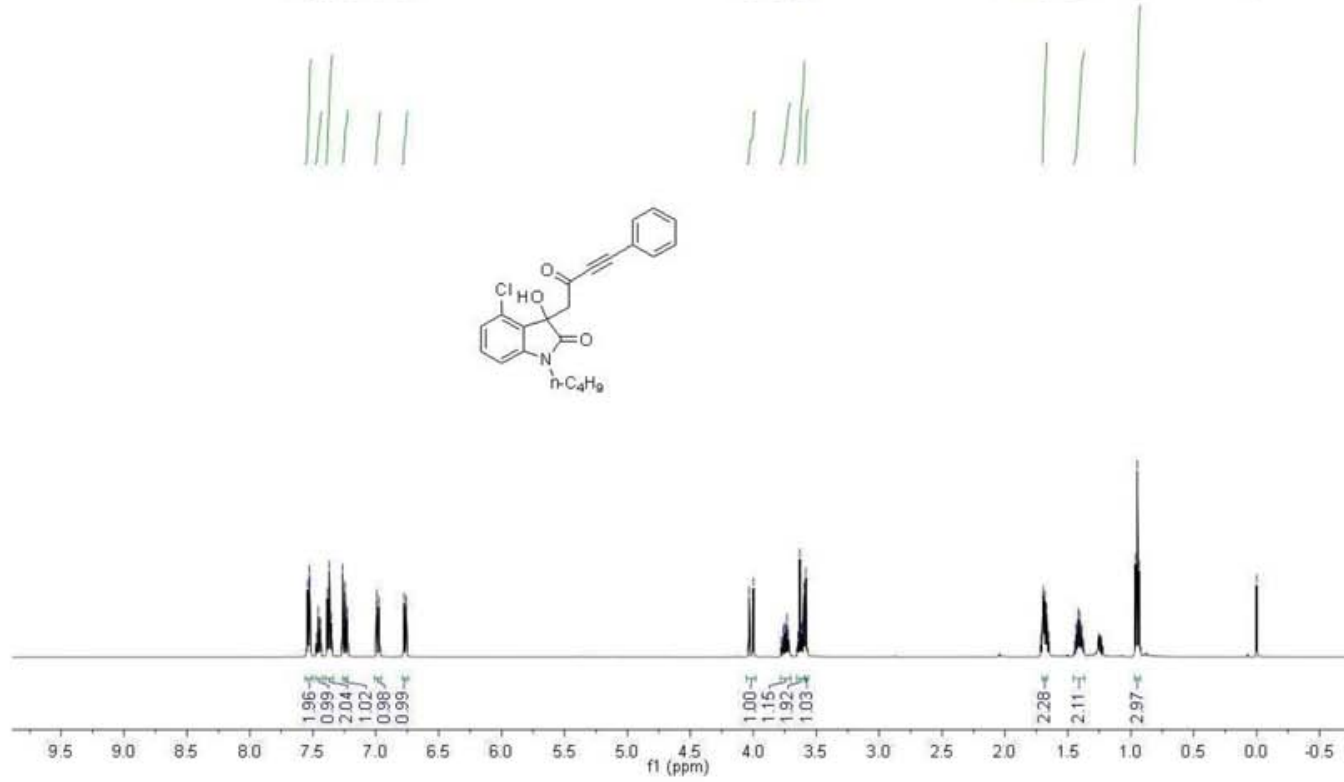

kev10113003

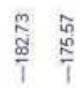
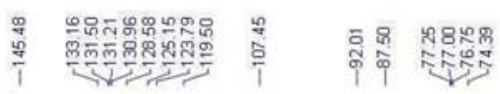

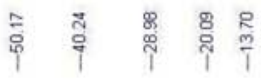
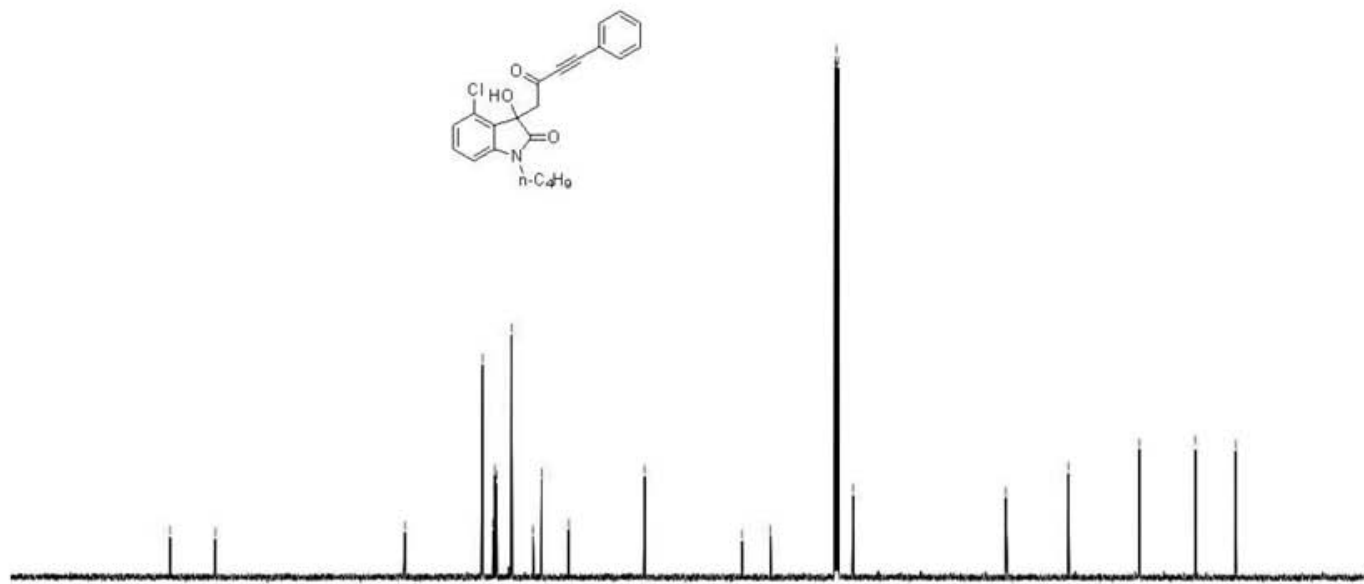

$\begin{array}{lllllllllll}200 & 190 & 180 & 170 & 160 & 150 & 140 & 130 & 120 & 110 & 100 \\ \text { f1 } & (\mathrm{pDN}) & & \end{array}$

Figure S2. ${ }^{1} \mathrm{H}$ NMR spectrum $\left(500 \mathrm{MHz} \mathrm{CDCl}_{3}\right)$ and ${ }^{13} \mathrm{C} \mathrm{NMR}$ spectrum $\left(126 \mathrm{MHz}^{\mathrm{CDCl}}{ }_{3}\right)$ of compound $4 \mathbf{b}$. 


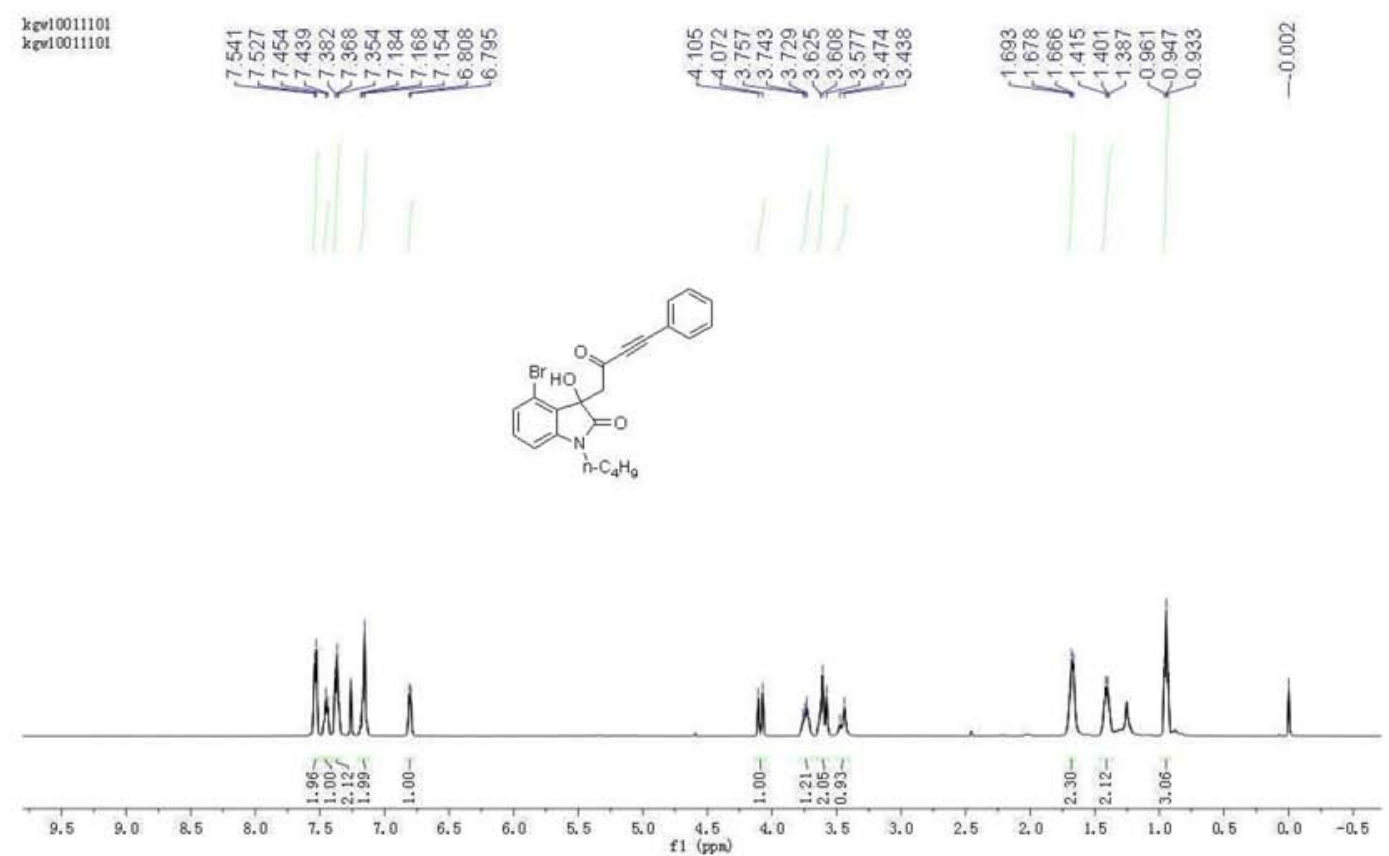

\begin{tabular}{|c|c|c|c|c|c|c|c|c|c|c|c|}
\hline $\begin{array}{l}\text { kzw10113001 } \\
\text { kzo10113001 }\end{array}$ & $\frac{\square}{\dddot{\Xi}}$ & $\begin{array}{l}\text { है } \\
\stackrel{1}{\uparrow}\end{array}$ & $\frac{\pi}{1}$ & 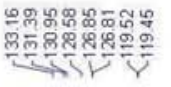 & के & 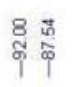 & 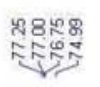 & $\begin{array}{l}8 \\
0 \\
0\end{array}$ & $\frac{\varrho}{\circ}$ & $\begin{array}{l}\mathscr{8} \\
\mathbb{7} \\
\text { Tे }\end{array}$ & $\frac{R}{7}$ \\
\hline
\end{tabular}

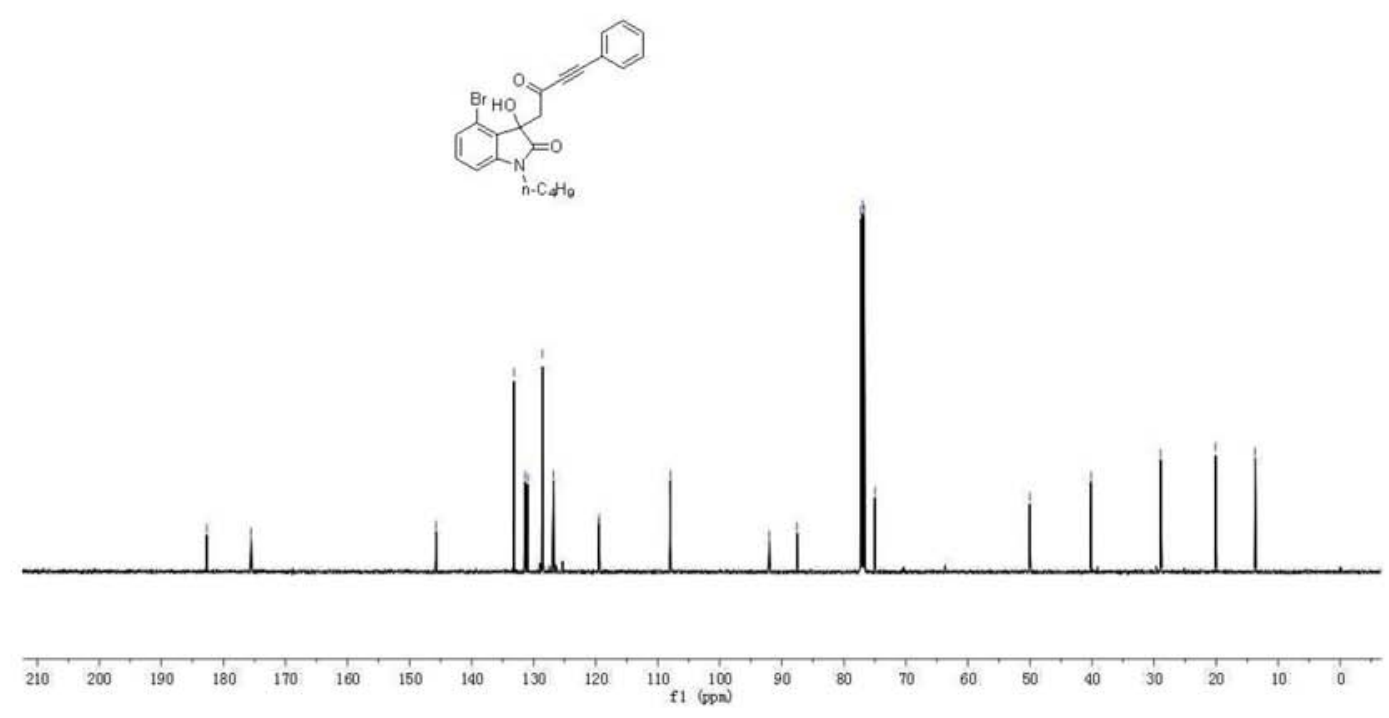

Figure S3. ${ }^{1} \mathrm{H}$ NMR spectrum $\left(500 \mathrm{MHz}, \mathrm{CDCl}_{3}\right)$ and ${ }^{13} \mathrm{C}$ NMR spectrum $\left(126 \mathrm{MHz}, \mathrm{CDCl}_{3}\right)$ of compound $4 \mathbf{c}$. 

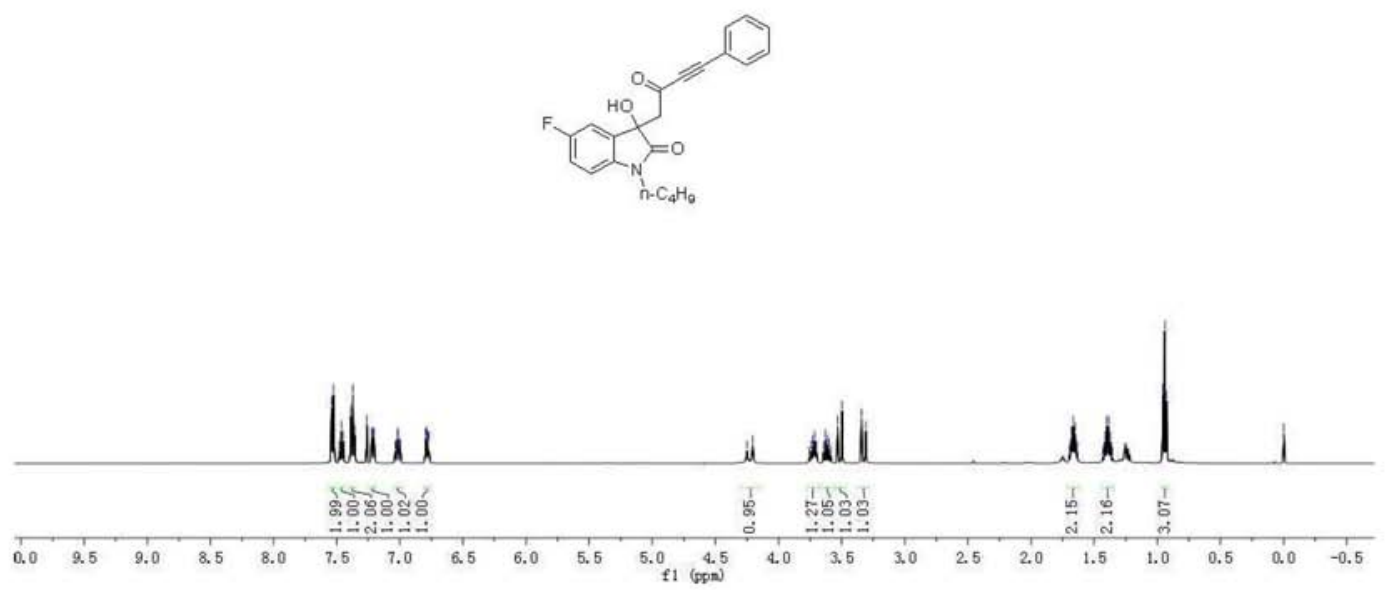

$k \approx 10112902$

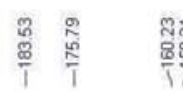
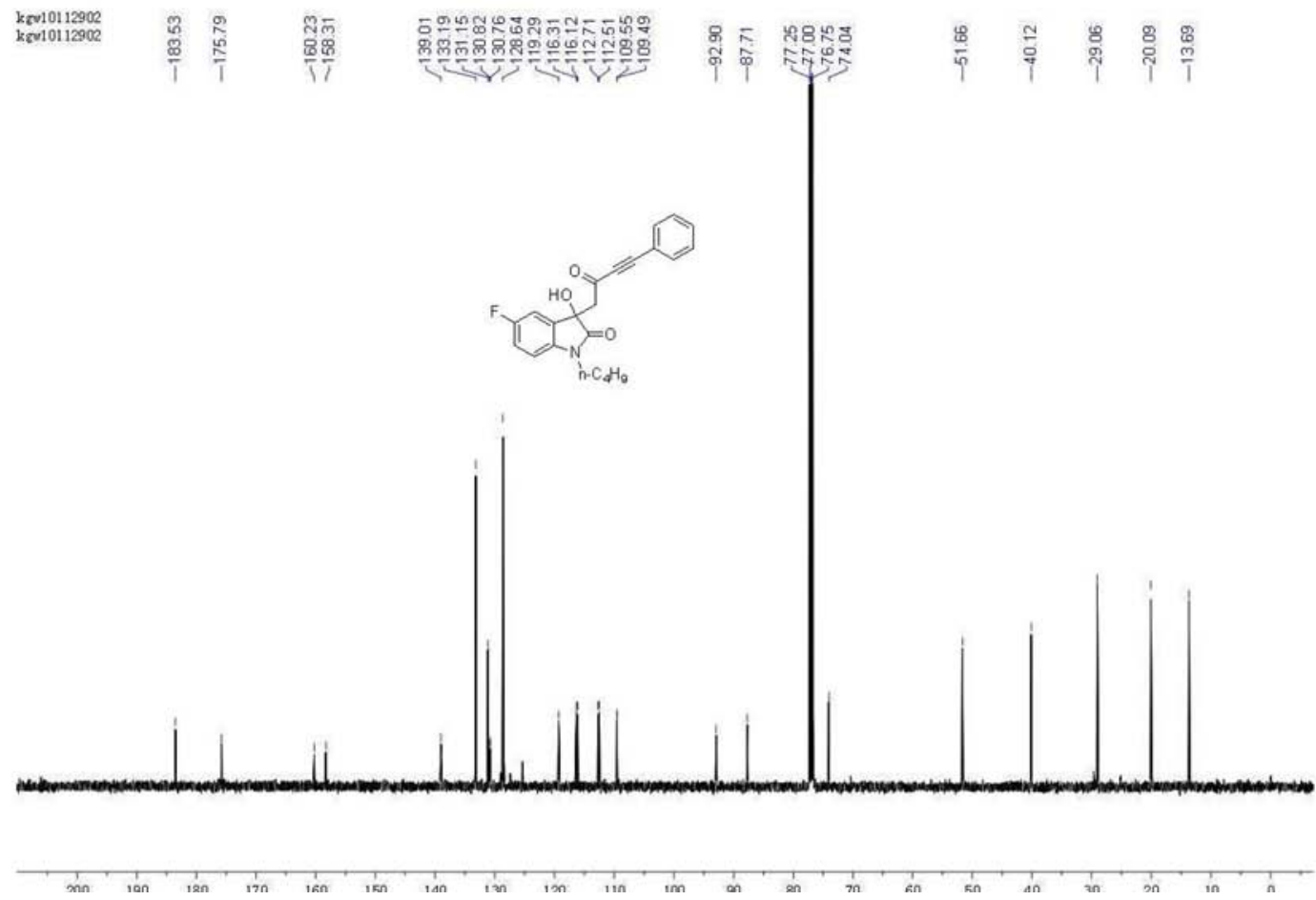

Figure S4. ${ }^{1} \mathrm{H}$ NMR spectrum $\left(500 \mathrm{MHz}, \mathrm{CDCl}_{3}\right)$ and ${ }^{13} \mathrm{C}$ NMR spectrum $\left(126 \mathrm{MHz}, \mathrm{CDCl}_{3}\right)$ of compound $4 \mathbf{e}$. 

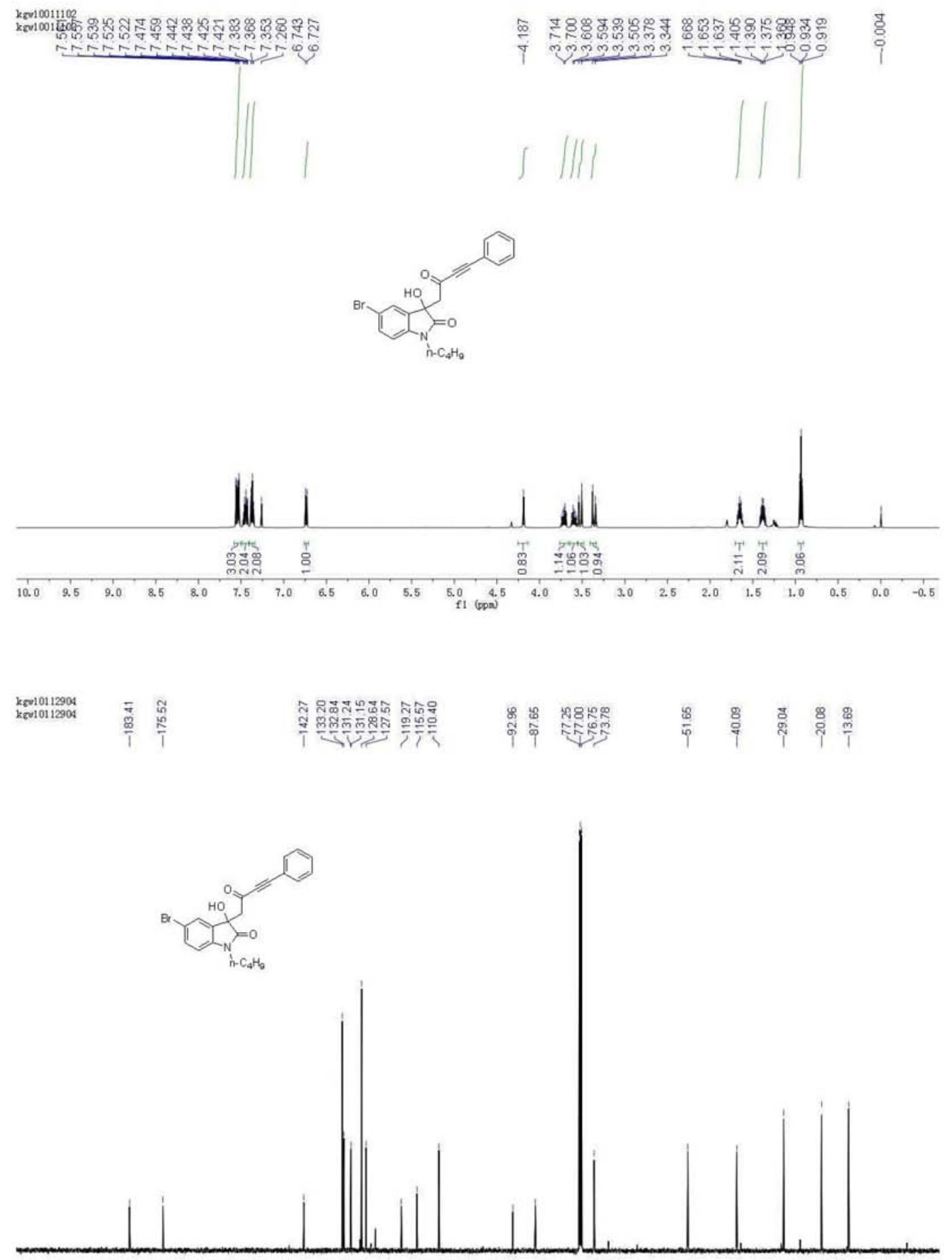

Figure S5. ${ }^{1} \mathrm{H}$ NMR spectrum $\left(500 \mathrm{MHz}, \mathrm{CDCl}_{3}\right)$ and ${ }^{13} \mathrm{C}$ NMR spectrum $\left(126 \mathrm{MHz}, \mathrm{CDCl}_{3}\right)$ of compound $\mathbf{4 g}$. 
kzon 10011106 kev10011106
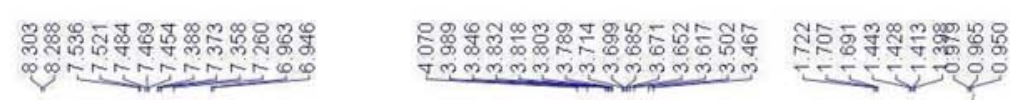

$\stackrel{2}{\circ}$

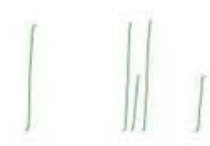<smiles>[I-]</smiles><smiles>[SiH2]</smiles>

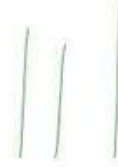

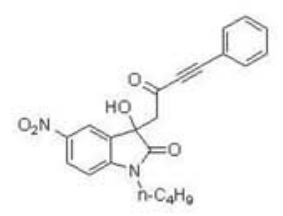

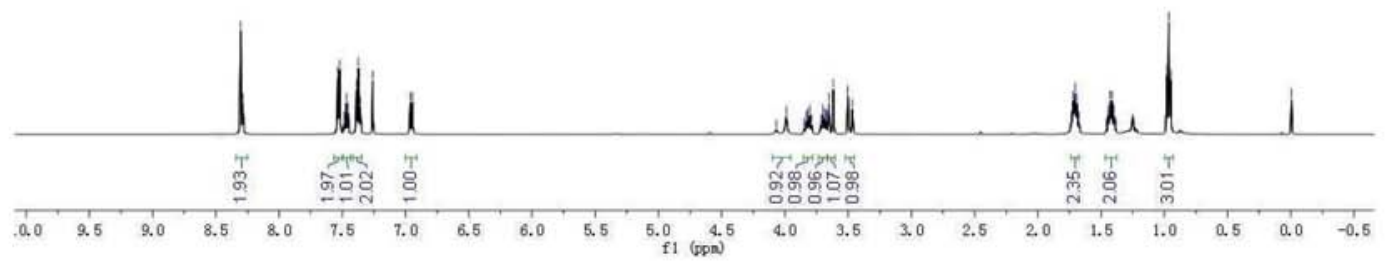

$k 20122807$
$k 20122807$

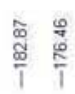

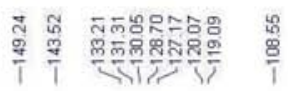

के लํ

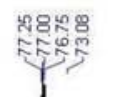

స ॠँ
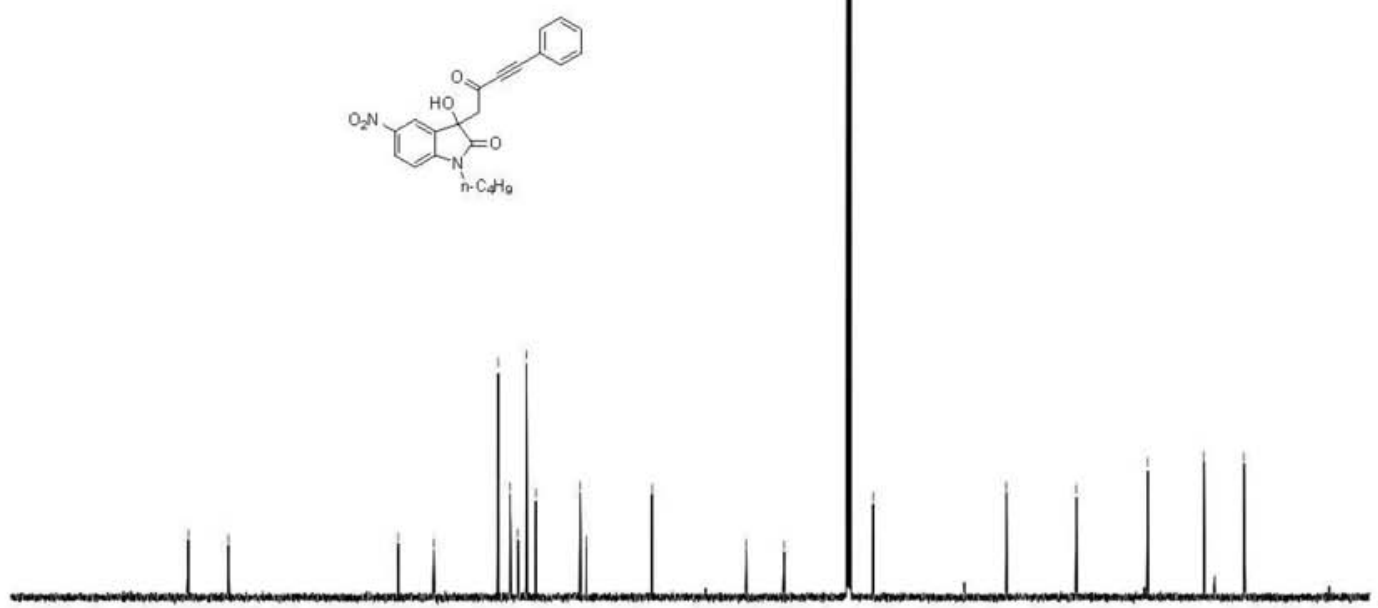

$\operatorname{Tin}$

Figure S6. ${ }^{1} \mathrm{H}$ NMR spectrum $\left(500 \mathrm{MHz}, \mathrm{CDCl}_{3}\right)$ and ${ }^{13} \mathrm{C} \mathrm{NMR}$ spectrum $\left(126 \mathrm{MHz}, \mathrm{CDCl}_{3}\right)$ of compound $4 \mathbf{h}$. 


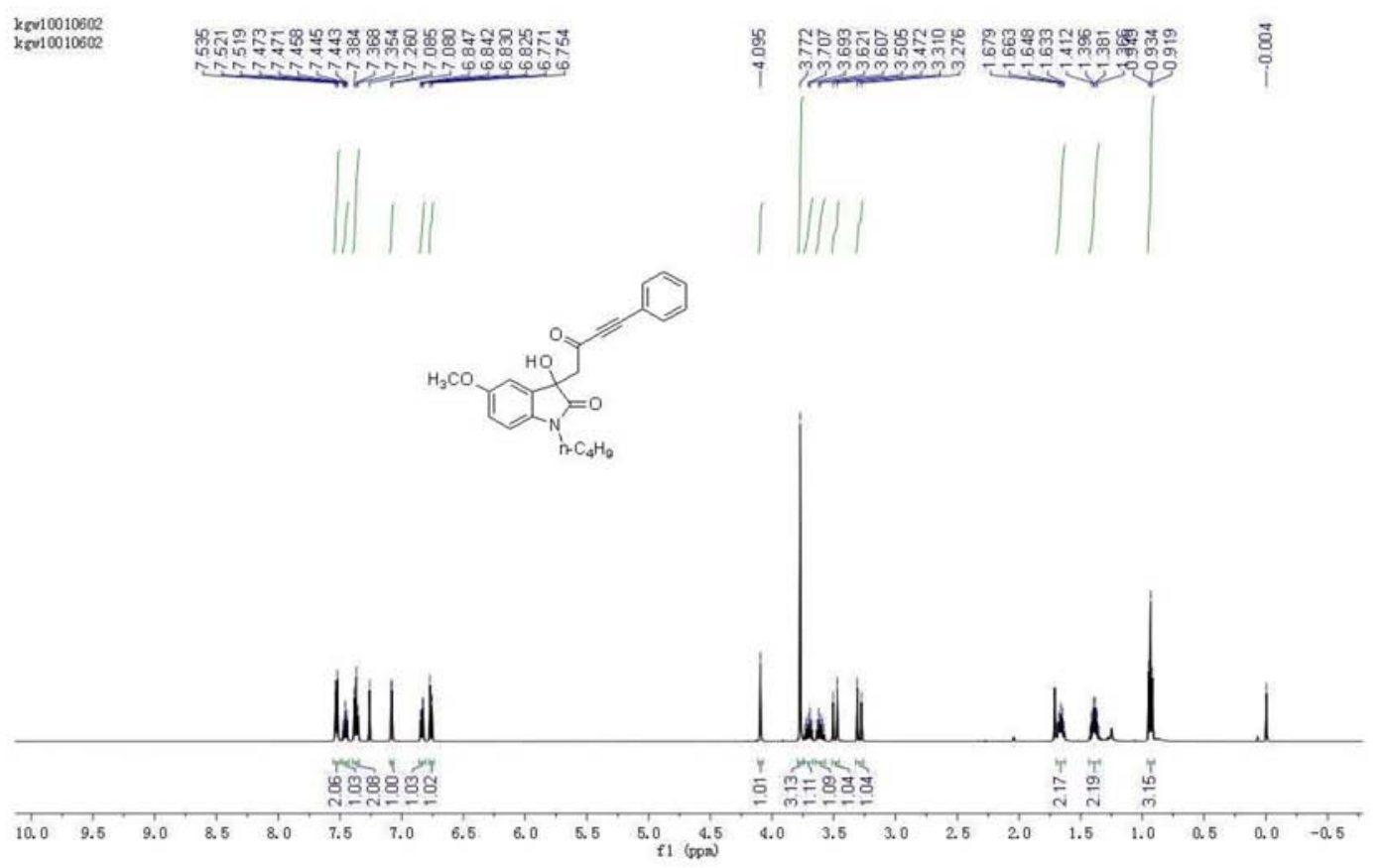

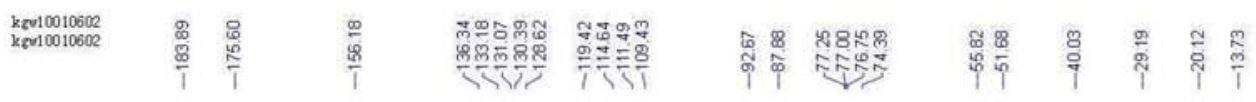
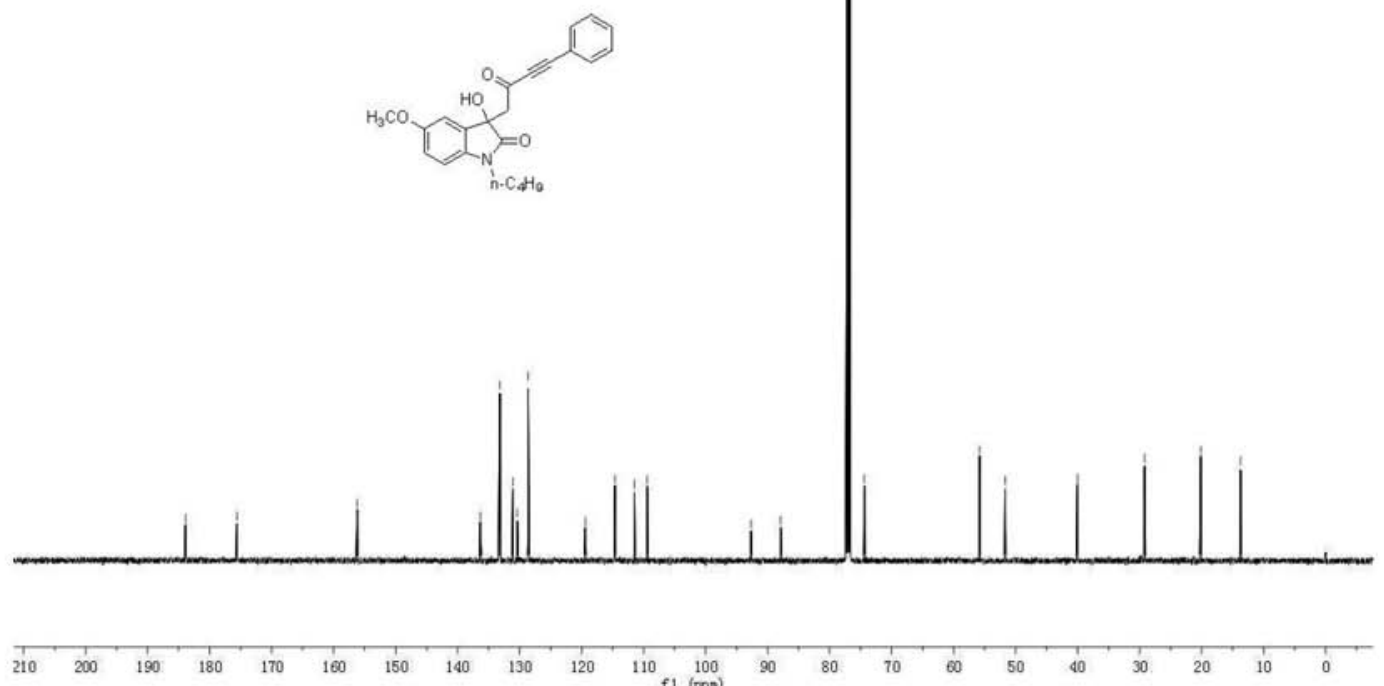

Figure S7. ${ }^{1} \mathrm{H}$ NMR spectrum $\left(500 \mathrm{MHz}, \mathrm{CDCl}_{3}\right)$ and ${ }^{13} \mathrm{C} \mathrm{NMR}$ spectrum $\left(126 \mathrm{MHz}, \mathrm{CDCl}_{3}\right)$ of compound $4 \mathbf{i}$. 

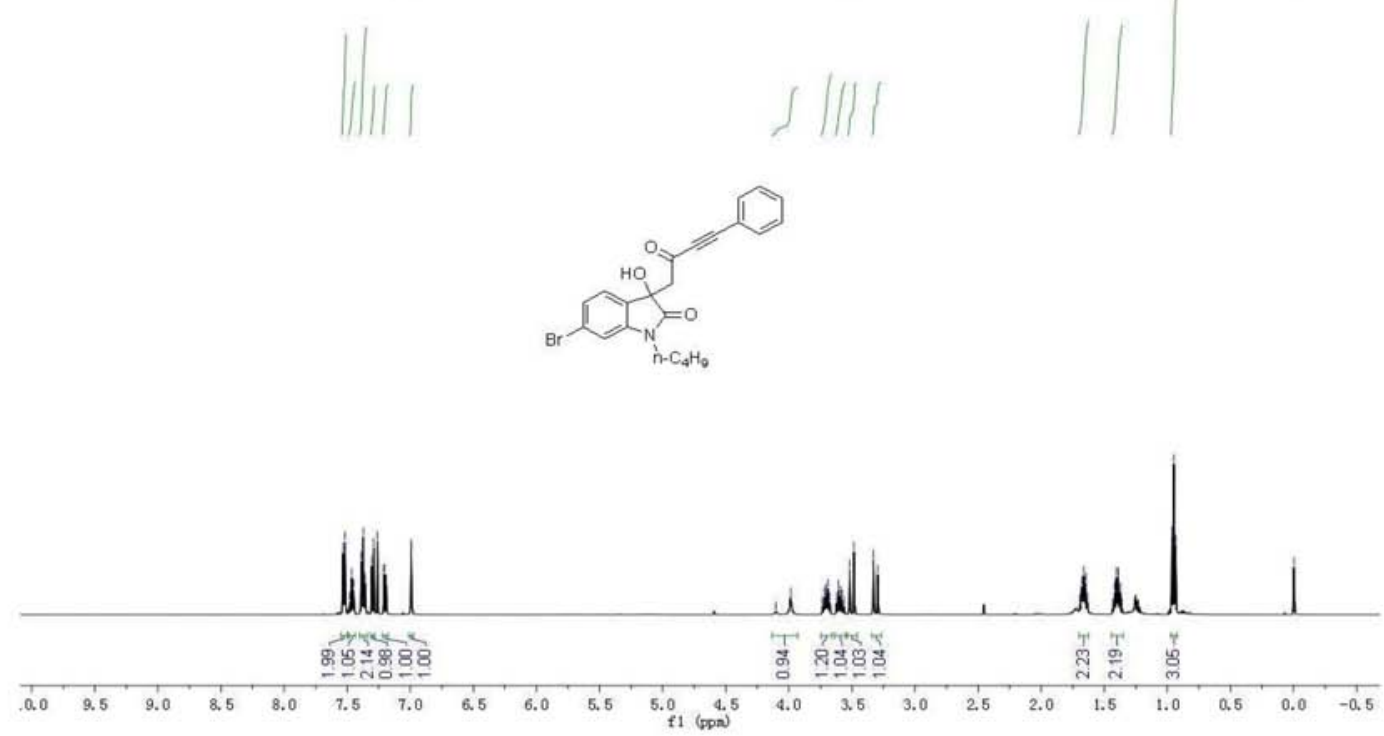

kev122803
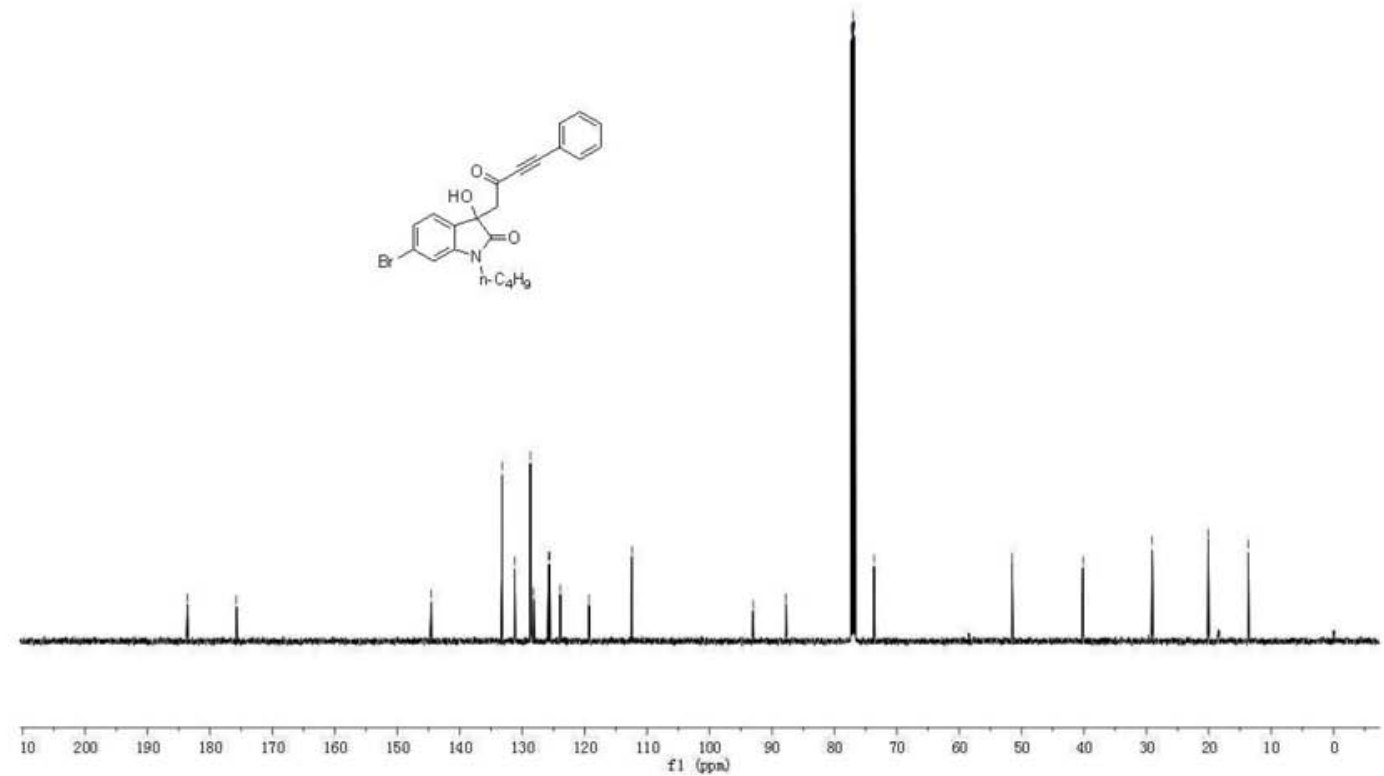

Figure S8. ${ }^{1} \mathrm{H}$ NMR spectrum $\left(500 \mathrm{MHz}, \mathrm{CDCl}_{3}\right)$ and ${ }^{13} \mathrm{C}$ NMR spectrum $\left(126 \mathrm{MHz}, \mathrm{CDCl}_{3}\right)$ compound of $\mathbf{4 j}$. 

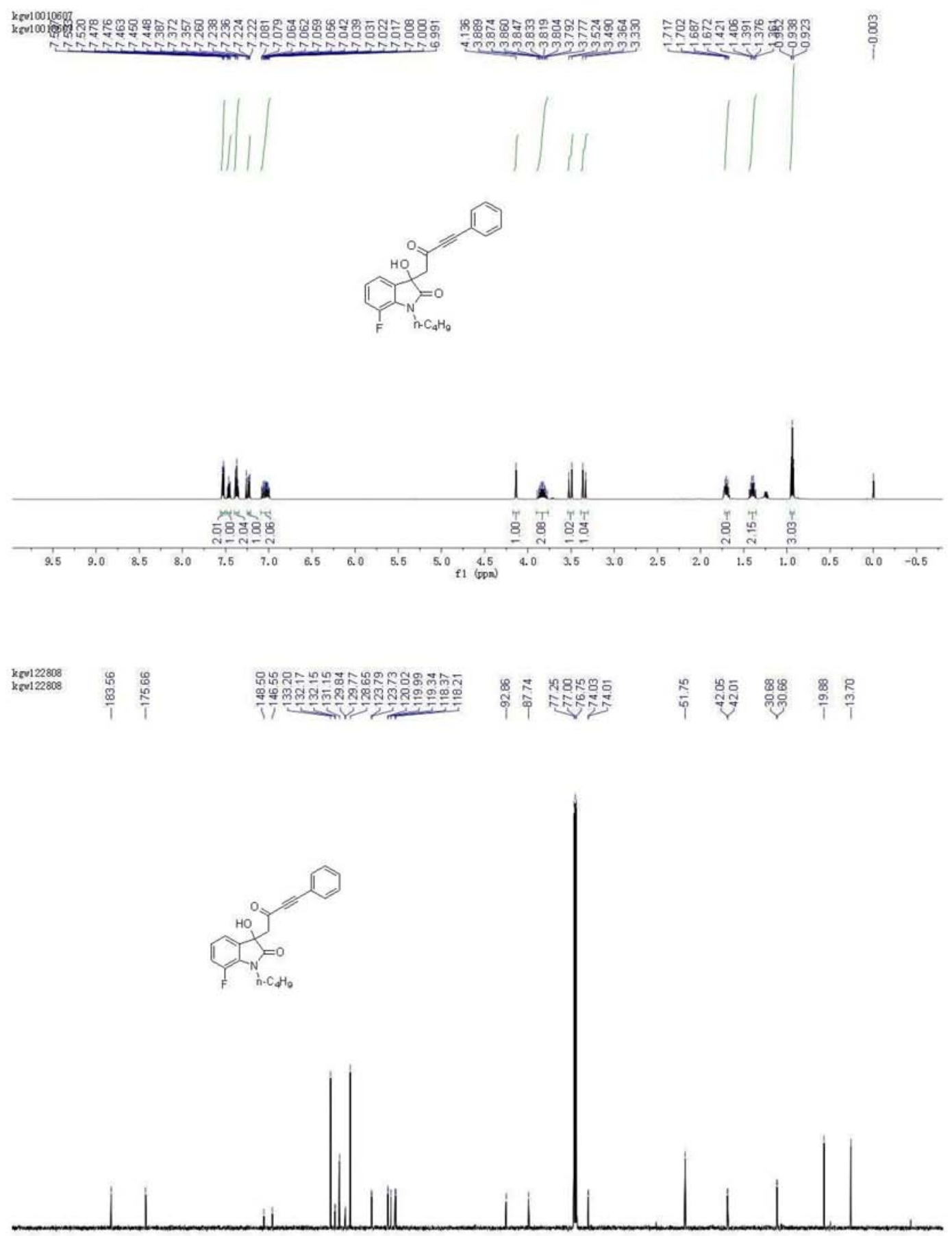

Figure S9. ${ }^{1} \mathrm{H}$ NMR spectrum $\left(500 \mathrm{MHz}, \mathrm{CDCl}_{3}\right)$ and ${ }^{13} \mathrm{C} \mathrm{NMR}$ spectrum $\left(126 \mathrm{MHz}, \mathrm{CDCl}_{3}\right)$ of compound $4 \mathbf{k}$. 

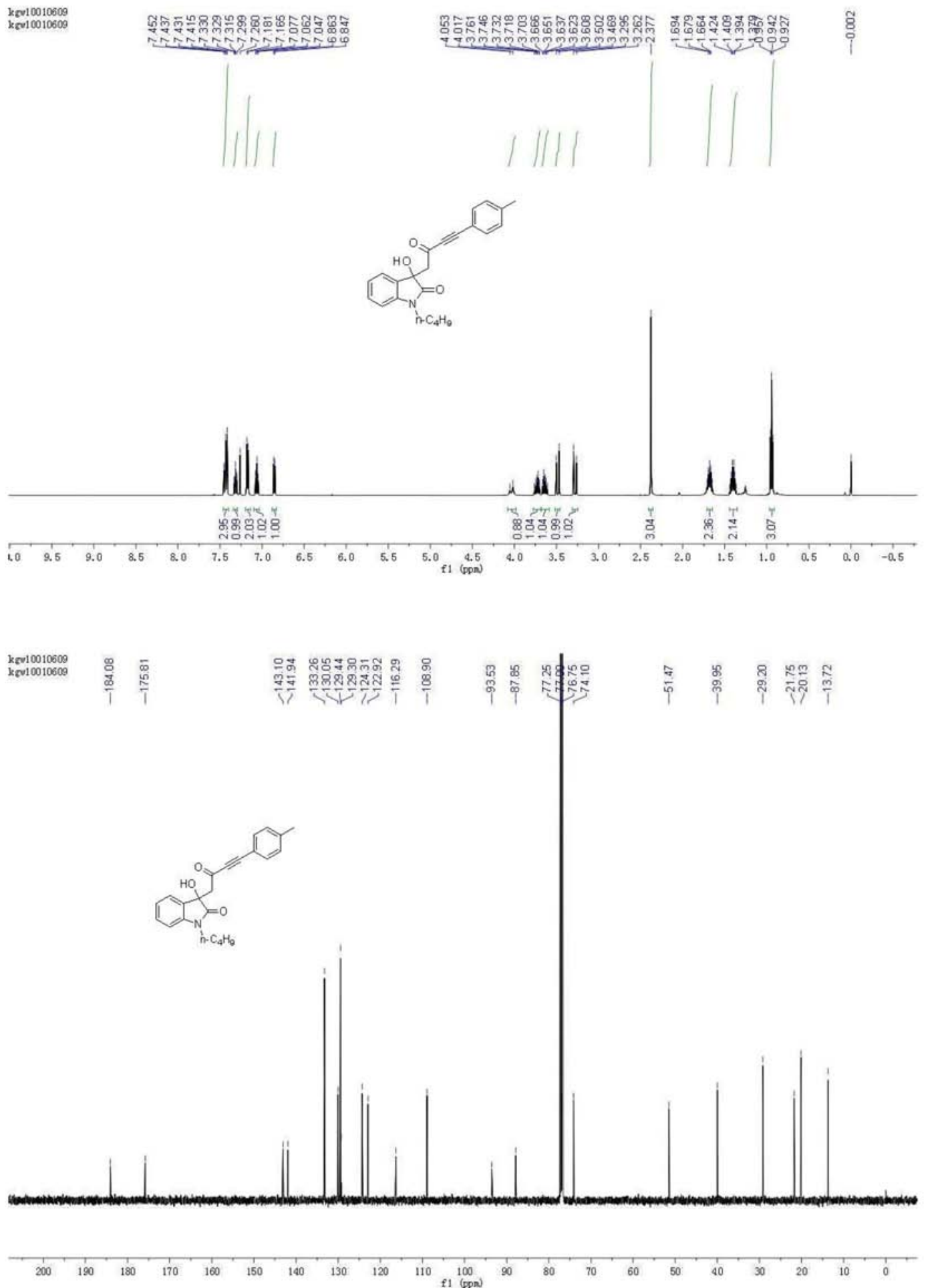

Figure S10. ${ }^{1} \mathrm{H}$ NMR spectrum $\left(500 \mathrm{MHz}, \mathrm{CDCl}_{3}\right)$ and ${ }^{13} \mathrm{C} \mathrm{NMR}$ spectrum $\left(126 \mathrm{MHz}, \mathrm{CDCl}_{3}\right)$ of compound $4 \mathbf{b a}$. 
kzo1001110? kev10011107

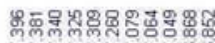
initising

$\| 11$

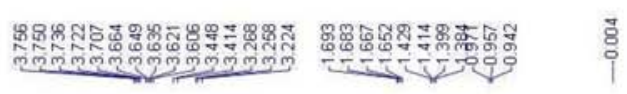

$1 / 1$

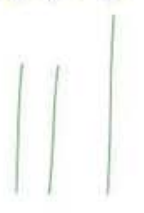

\section{i}

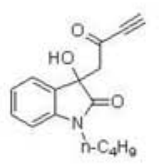

kev10010610 kख 10010610
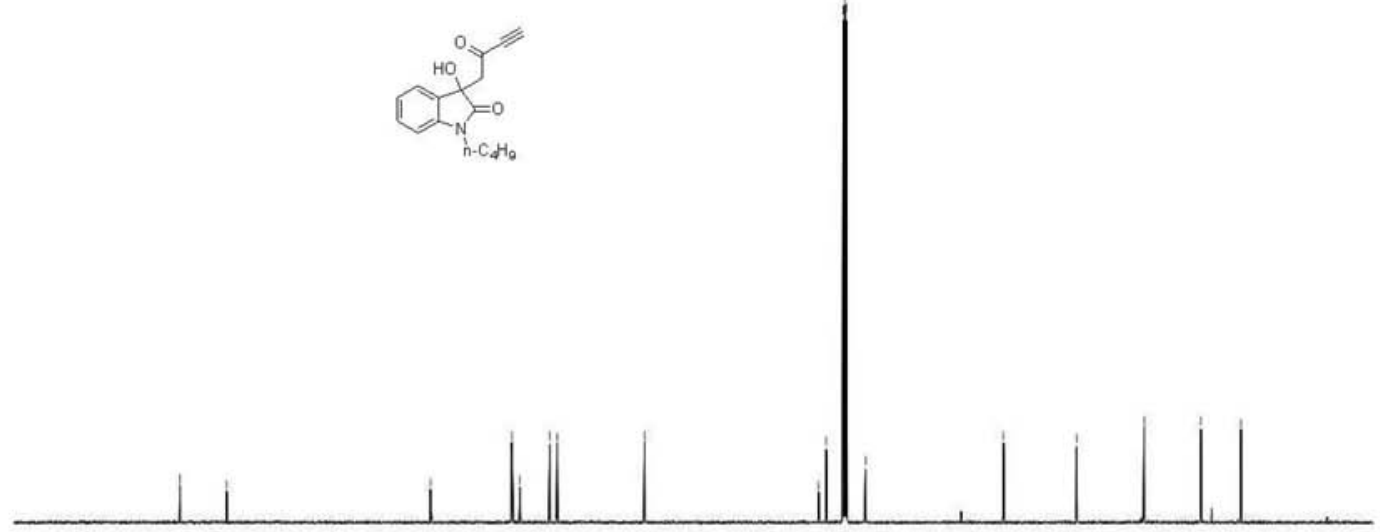

Figure S11. ${ }^{1} \mathrm{H}$ NMR spectrum $\left(500 \mathrm{MHz}, \mathrm{CDCl}_{3}\right)$ and ${ }^{13} \mathrm{C} \mathrm{NMR}$ spectrum $\left(126 \mathrm{MHz}, \mathrm{CDCl}_{3}\right.$ ) of compound $\mathbf{4} \mathbf{c a}$. 

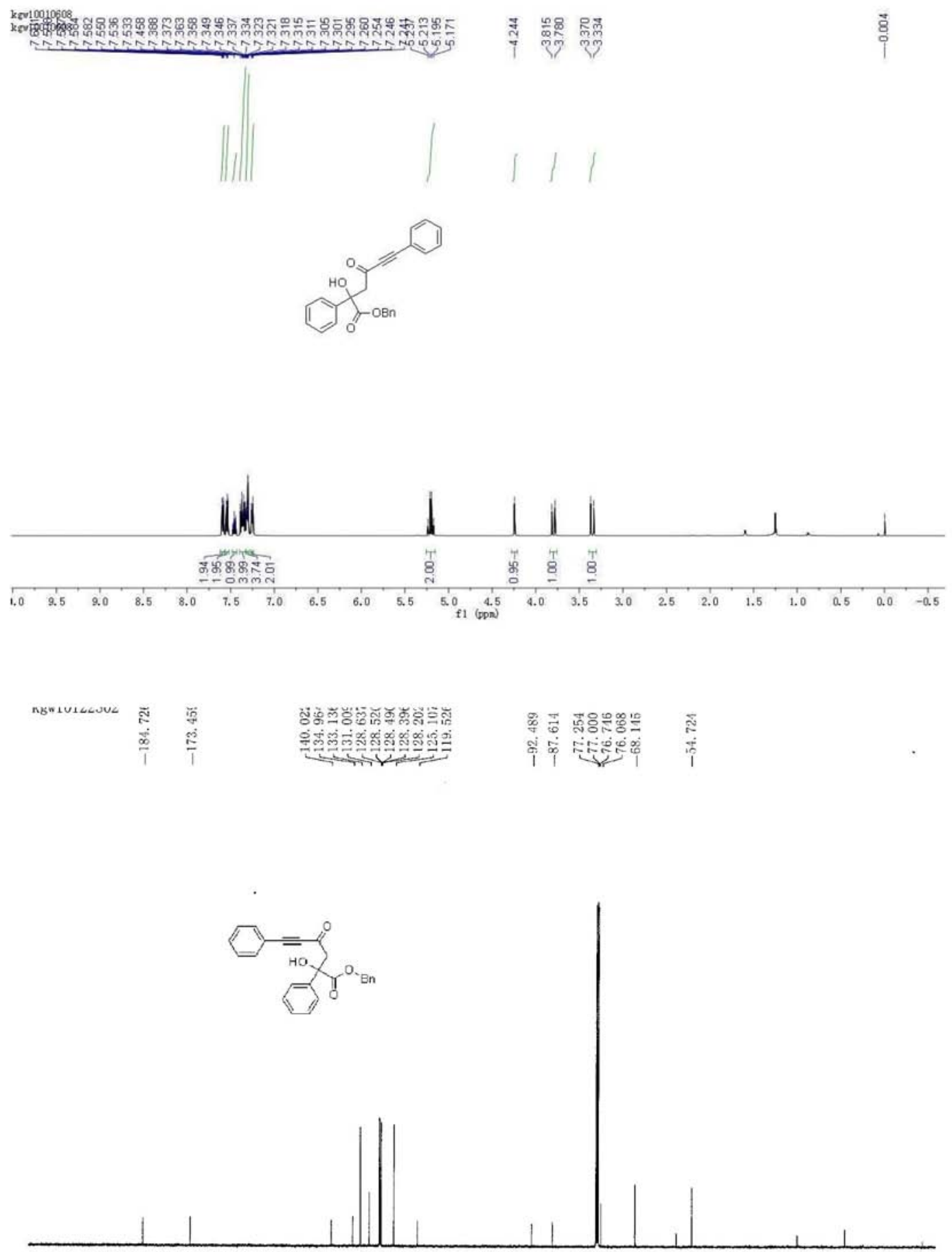

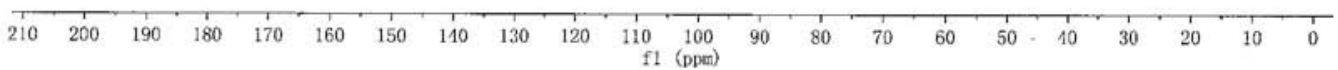

Figure S12. ${ }^{1} \mathrm{H}$ NMR spectrum $\left(500 \mathrm{MHz}, \mathrm{CDCl}_{3}\right)$ and ${ }^{13} \mathrm{C}$ NMR spectrum $\left(126 \mathrm{MHz}, \mathrm{CDCl}_{3}\right.$ ) of compound 41. 

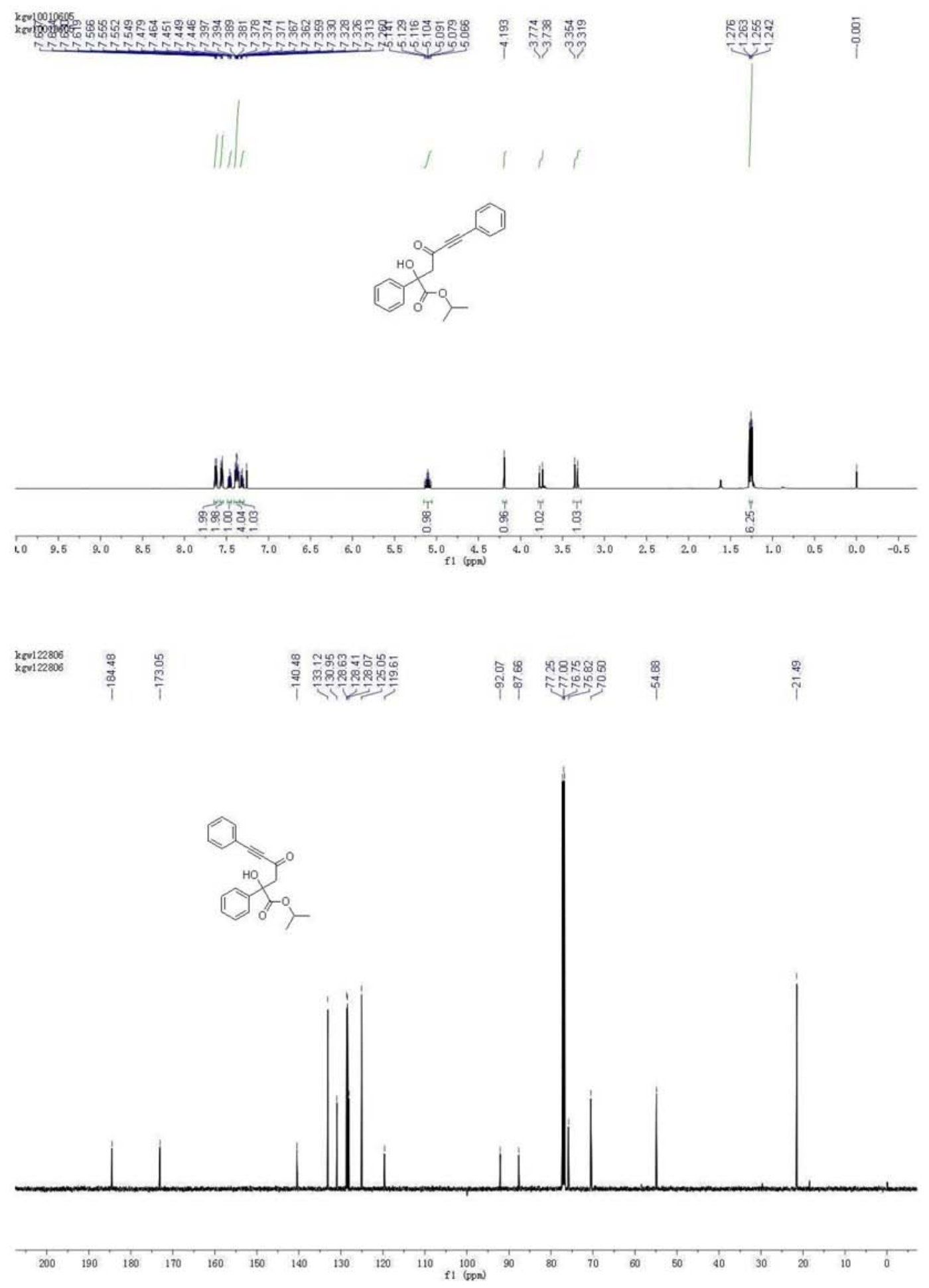

Figure S13. ${ }^{1} \mathrm{H}$ NMR spectrum $\left(500 \mathrm{MHz}, \mathrm{CDCl}_{3}\right)$ and ${ }^{13} \mathrm{C} \mathrm{NMR}$ spectrum $\left(126 \mathrm{MHz}, \mathrm{CDCl}_{3}\right.$ ) of compound $\mathbf{4 m}$. 


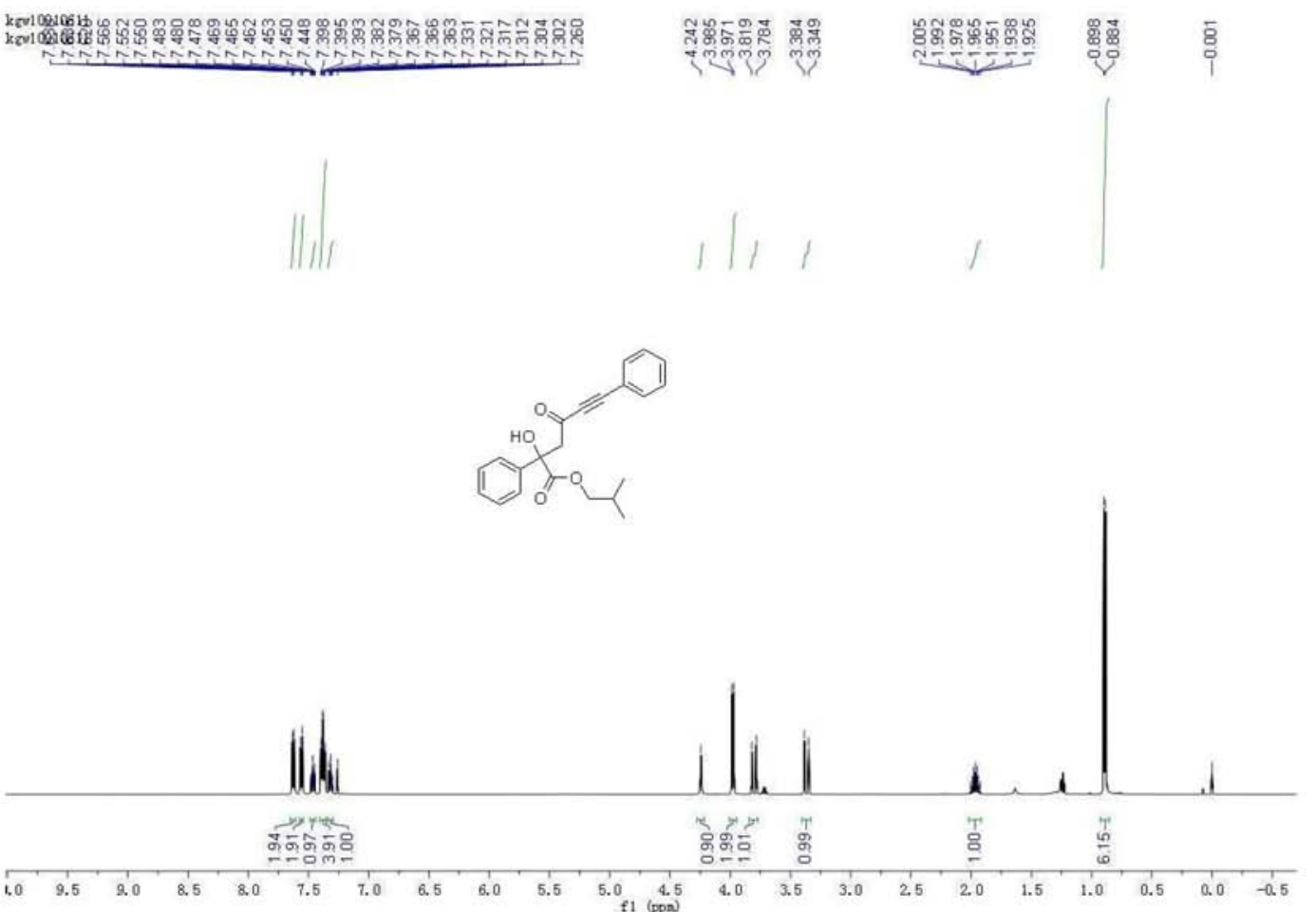

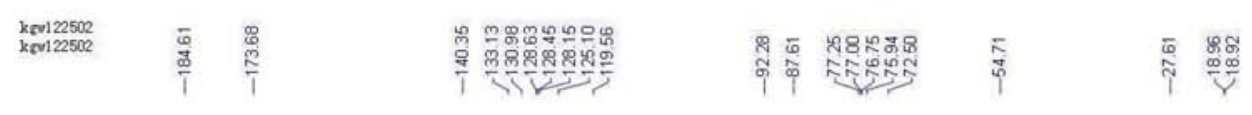
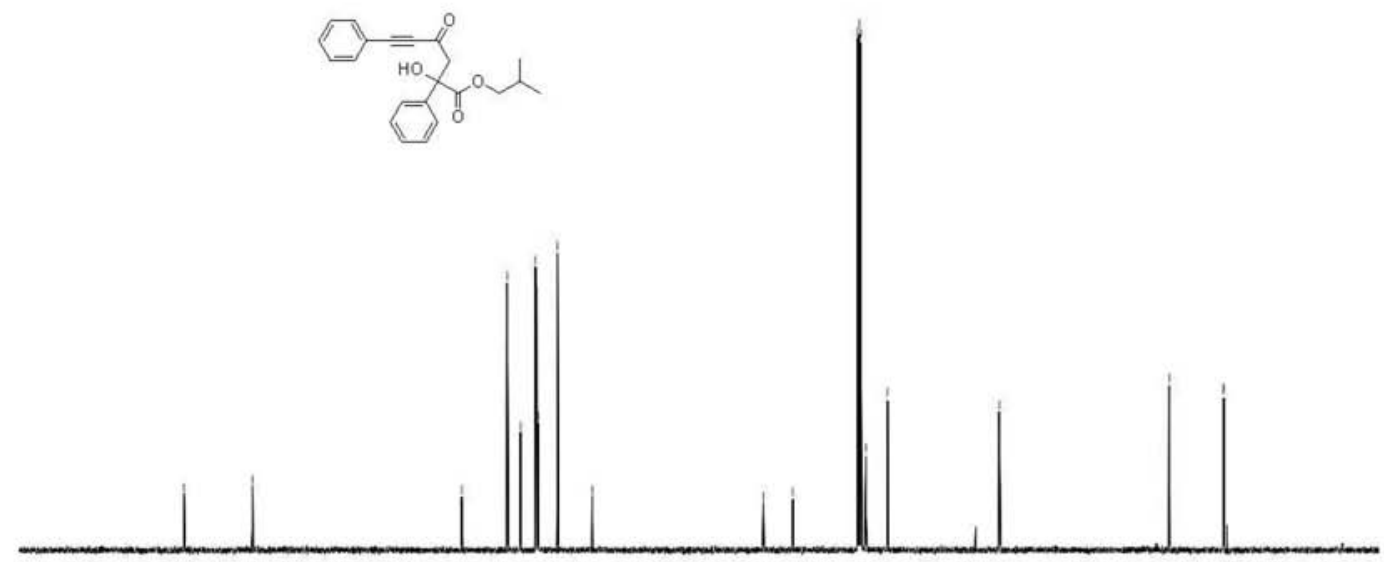

Figure S14. ${ }^{1} \mathrm{H}$ NMR spectrum $\left(500 \mathrm{MHz}, \mathrm{CDCl}_{3}\right)$ and ${ }^{13} \mathrm{C} \mathrm{NMR}$ spectrum $\left(126 \mathrm{MHz}, \mathrm{CDCl}_{3}\right)$ of compound $\mathbf{4 n}$. 


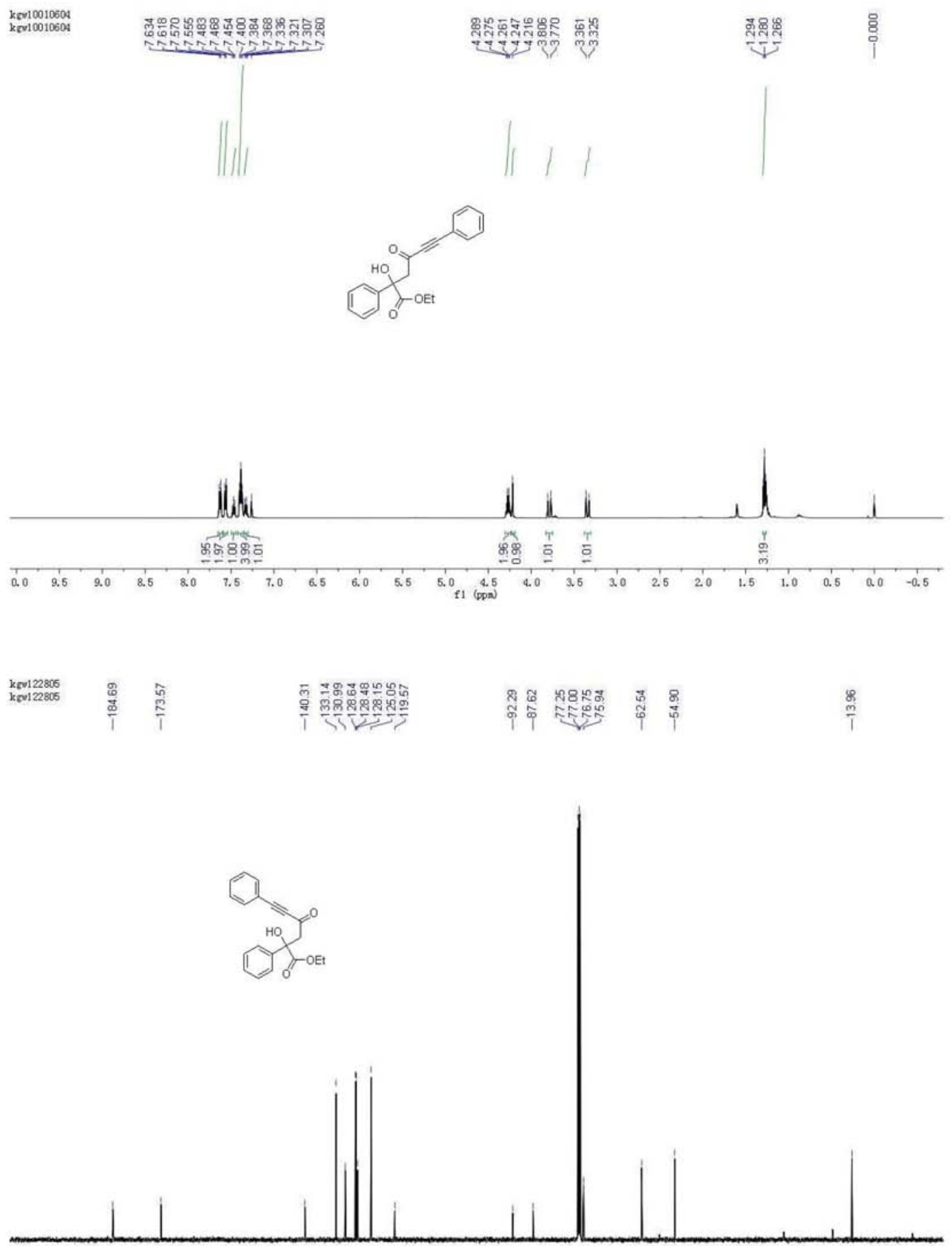



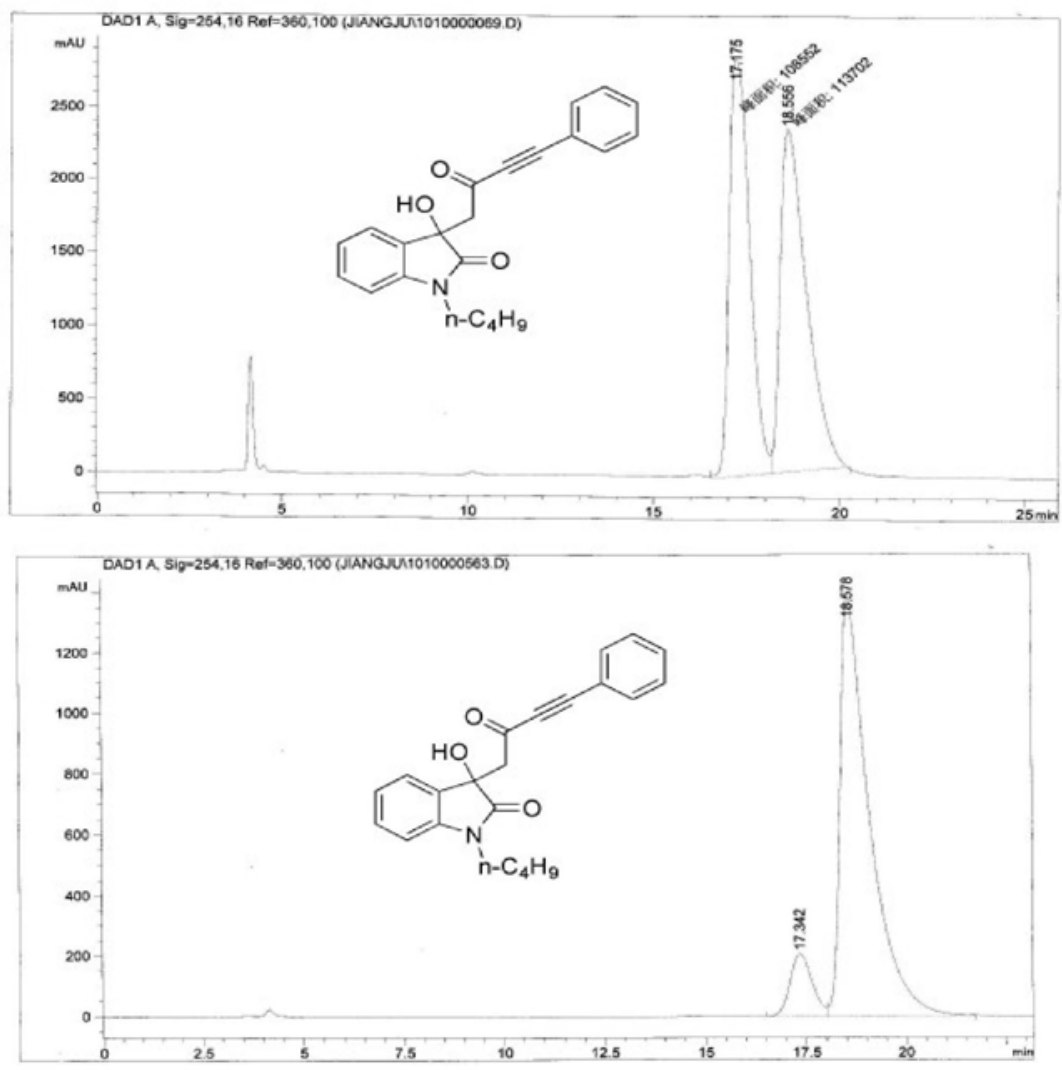

Figure S16. HPLC of compound 4a.
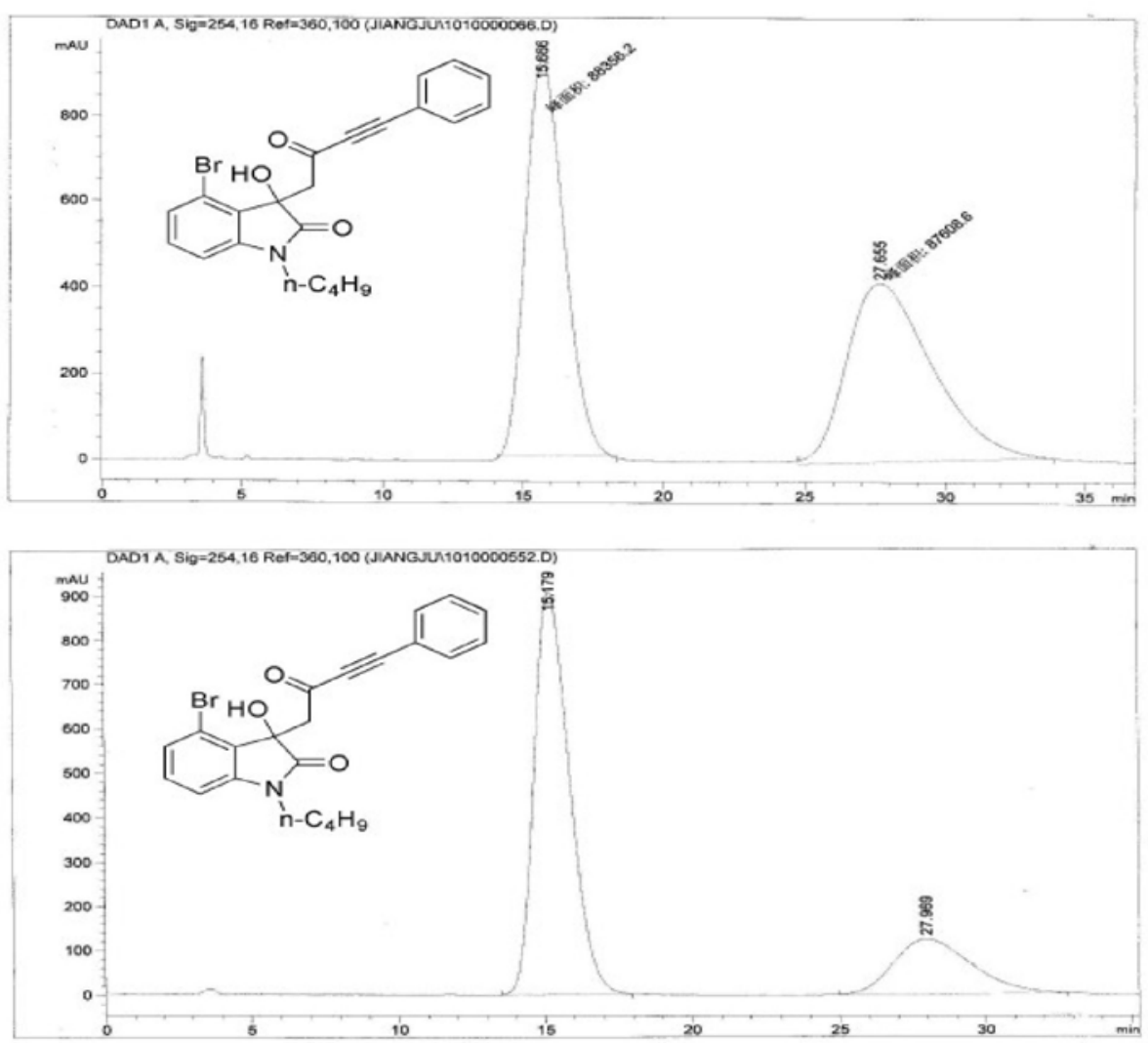

Figure S17. HPLC of compound 4c. 
Vol. 23, No. 1, 2012

Kang et al.

S21
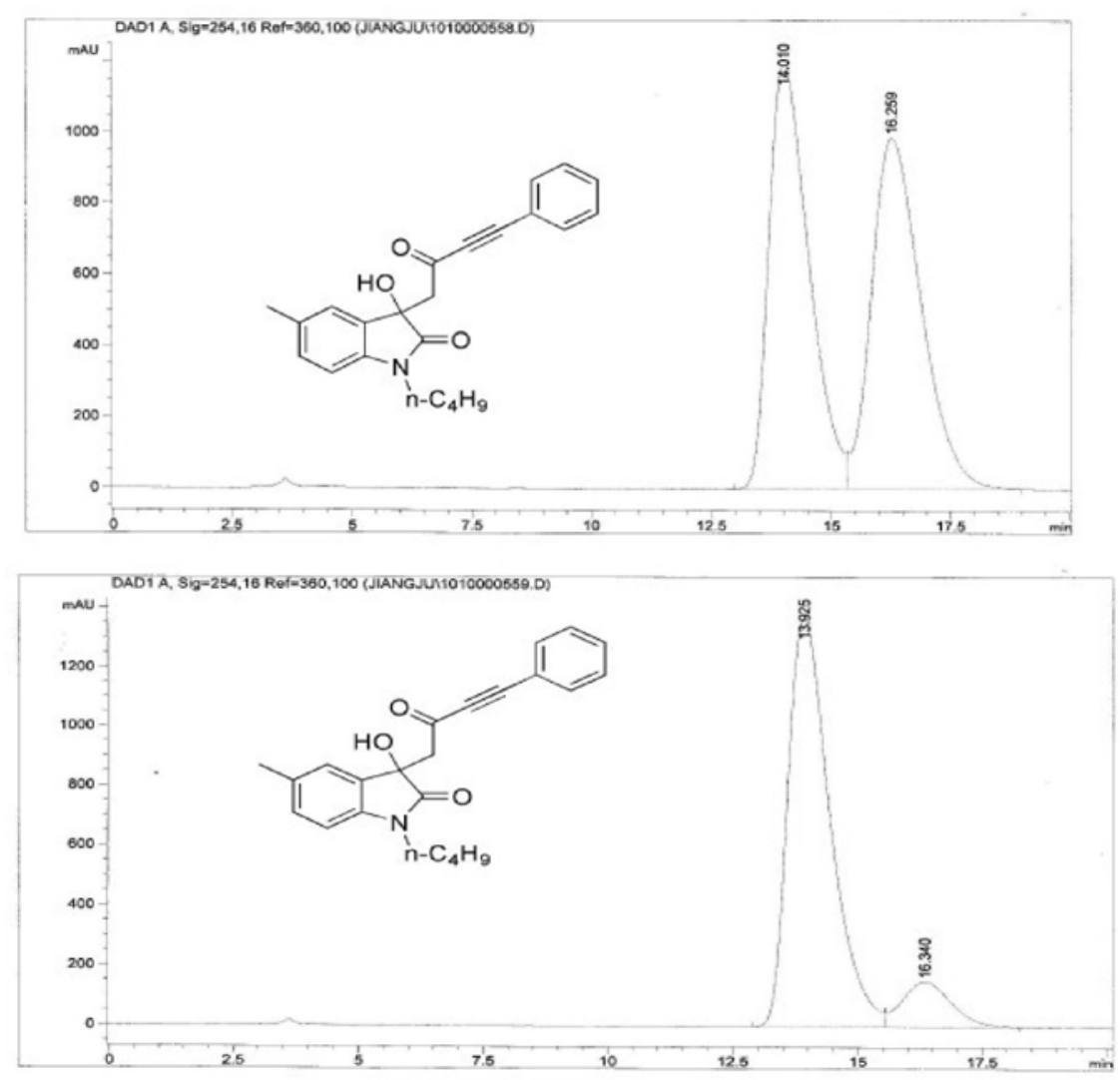

Figure S18. HPLC of compound 4d.
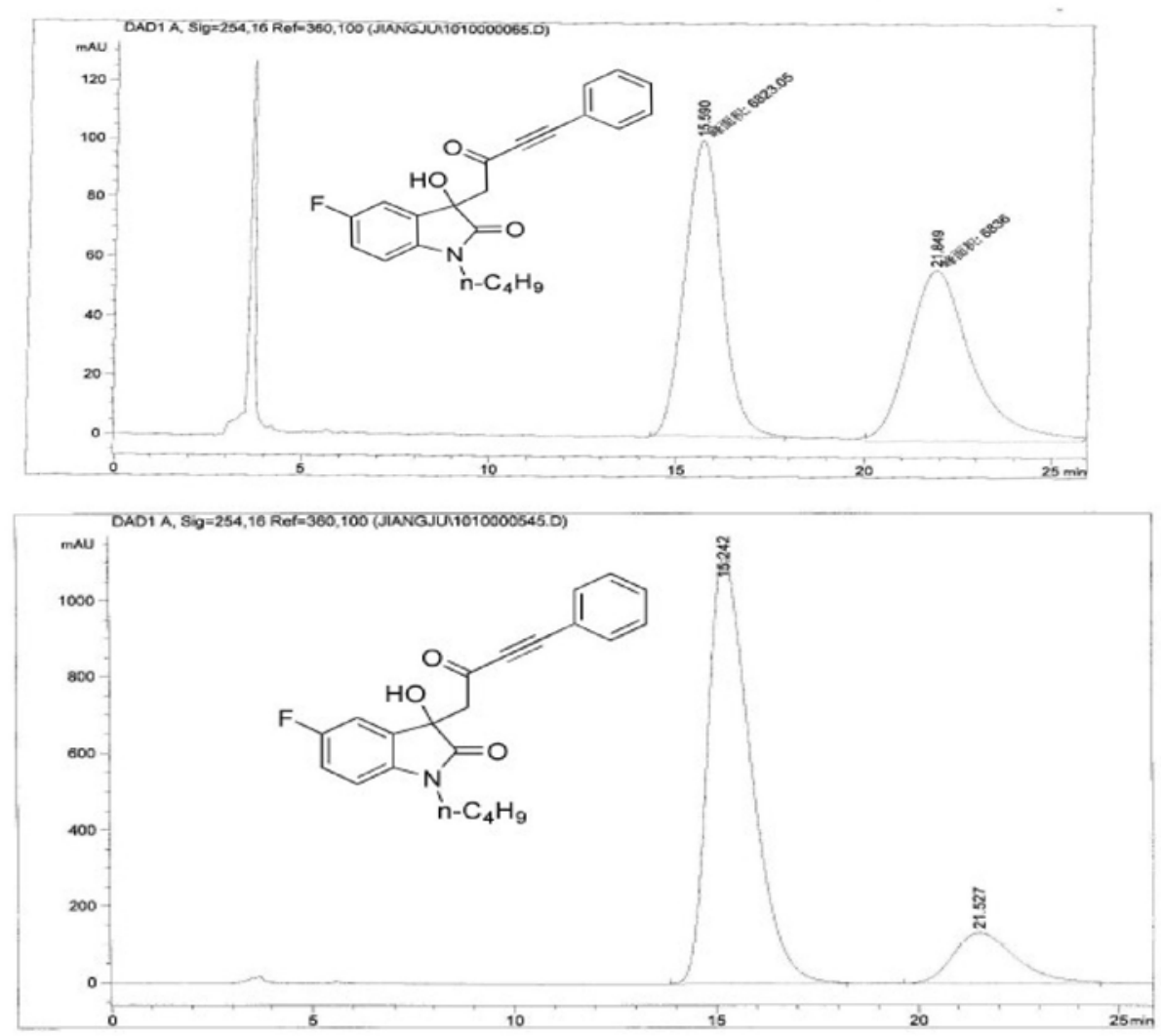

Figure S19. HPLC of compound 4e. 

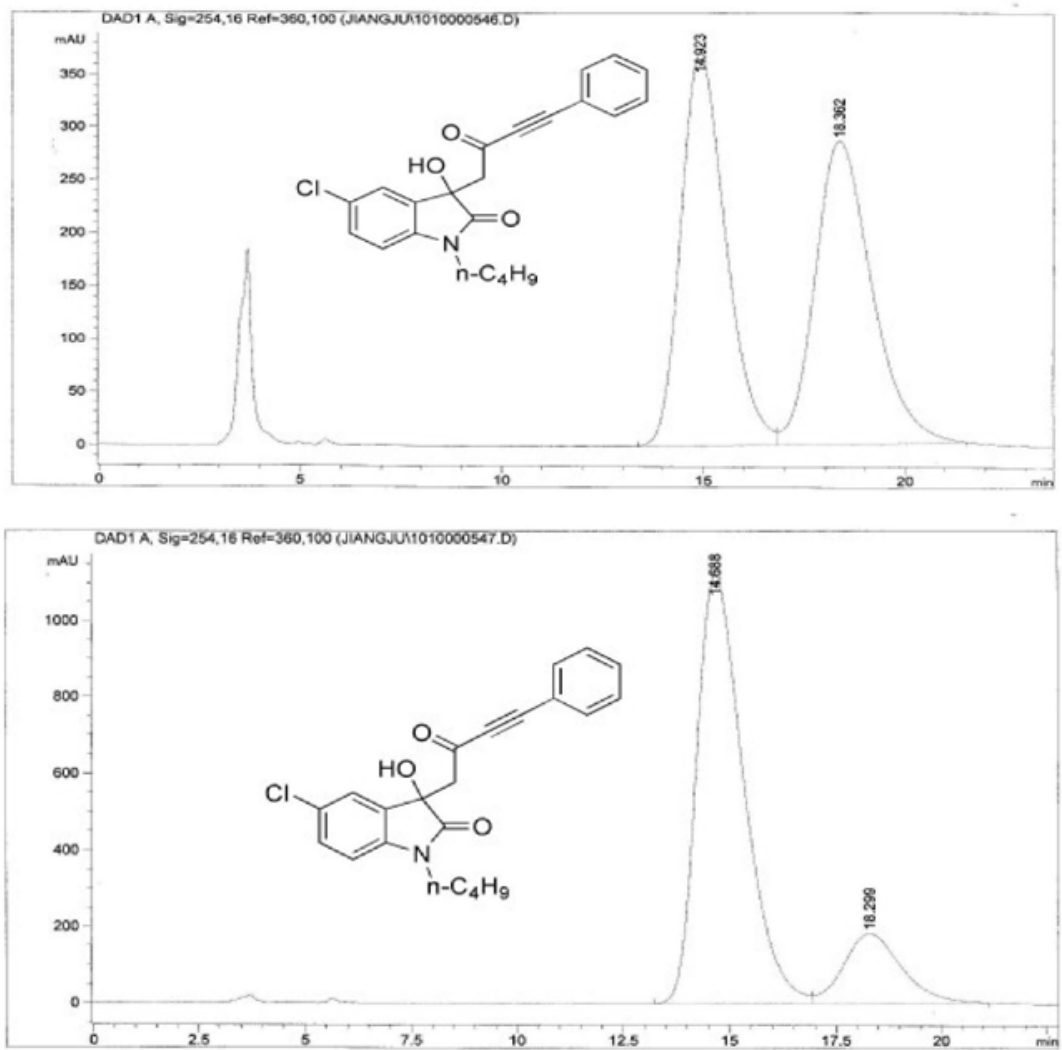

Figure S20. HPLC of compound $4 f$
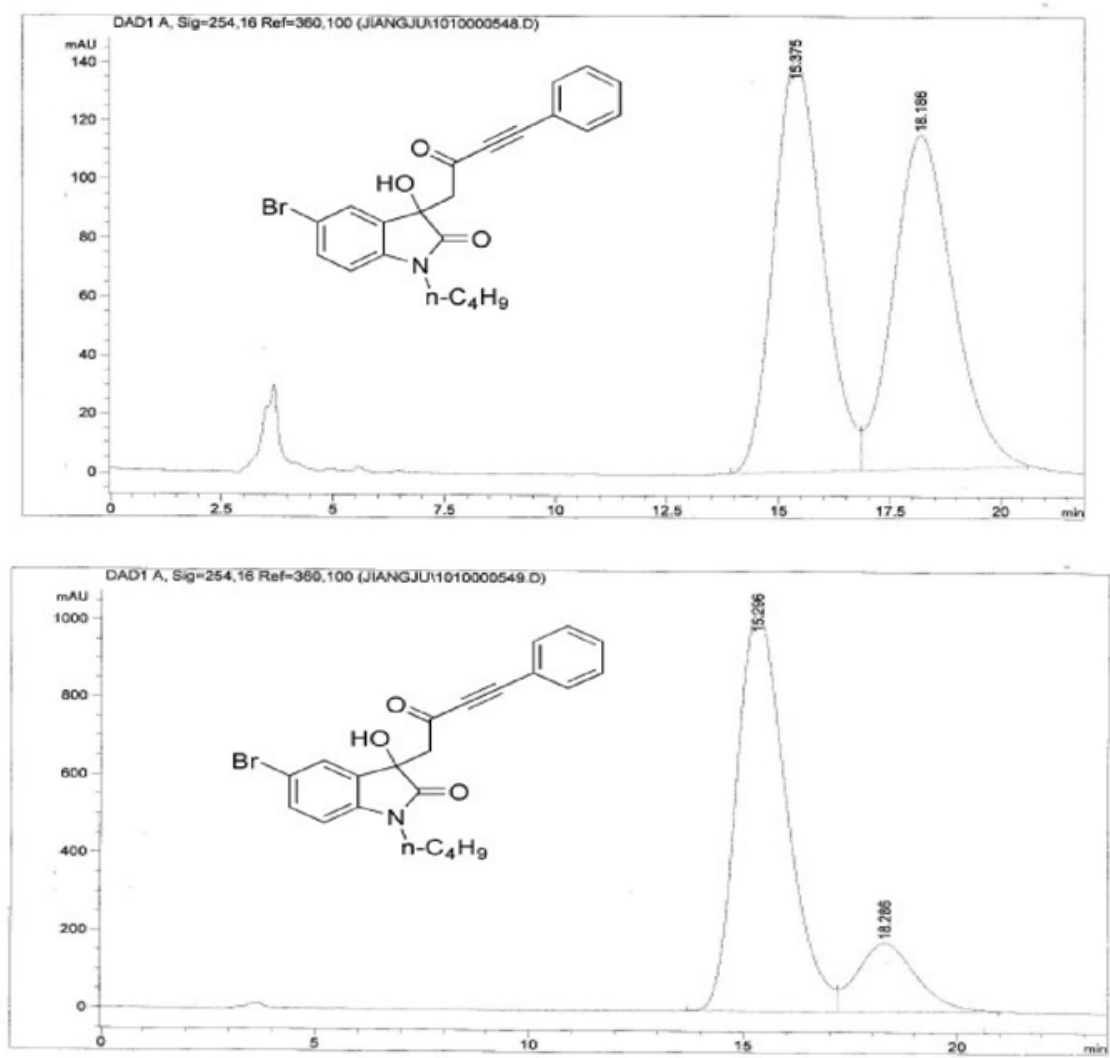

Figure S21. HPLC of compound $\mathbf{4 g}$ 
Vol. 23, No. 1, 2012

Kang et al.

S23
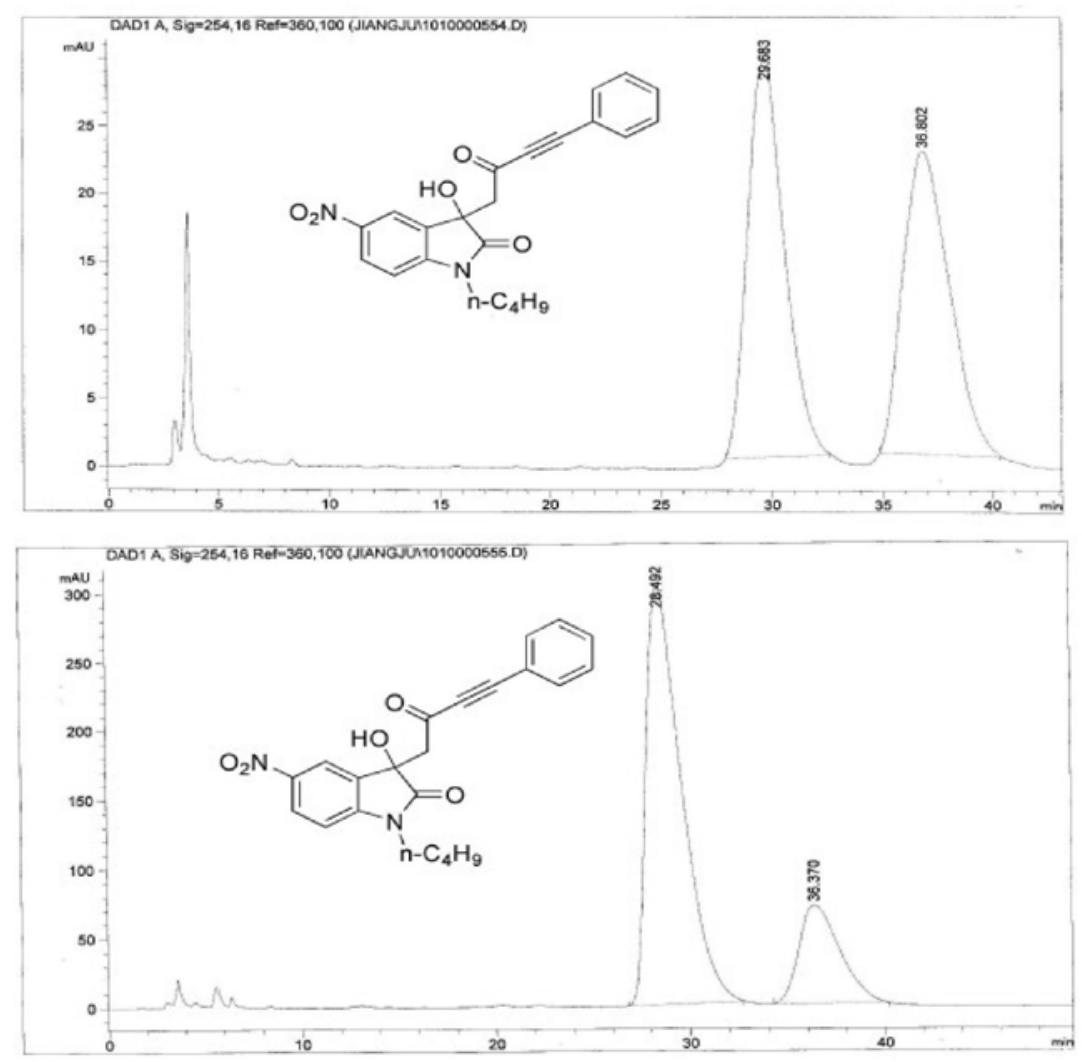

Figure S22. HPLC of compound $\mathbf{4 h}$.
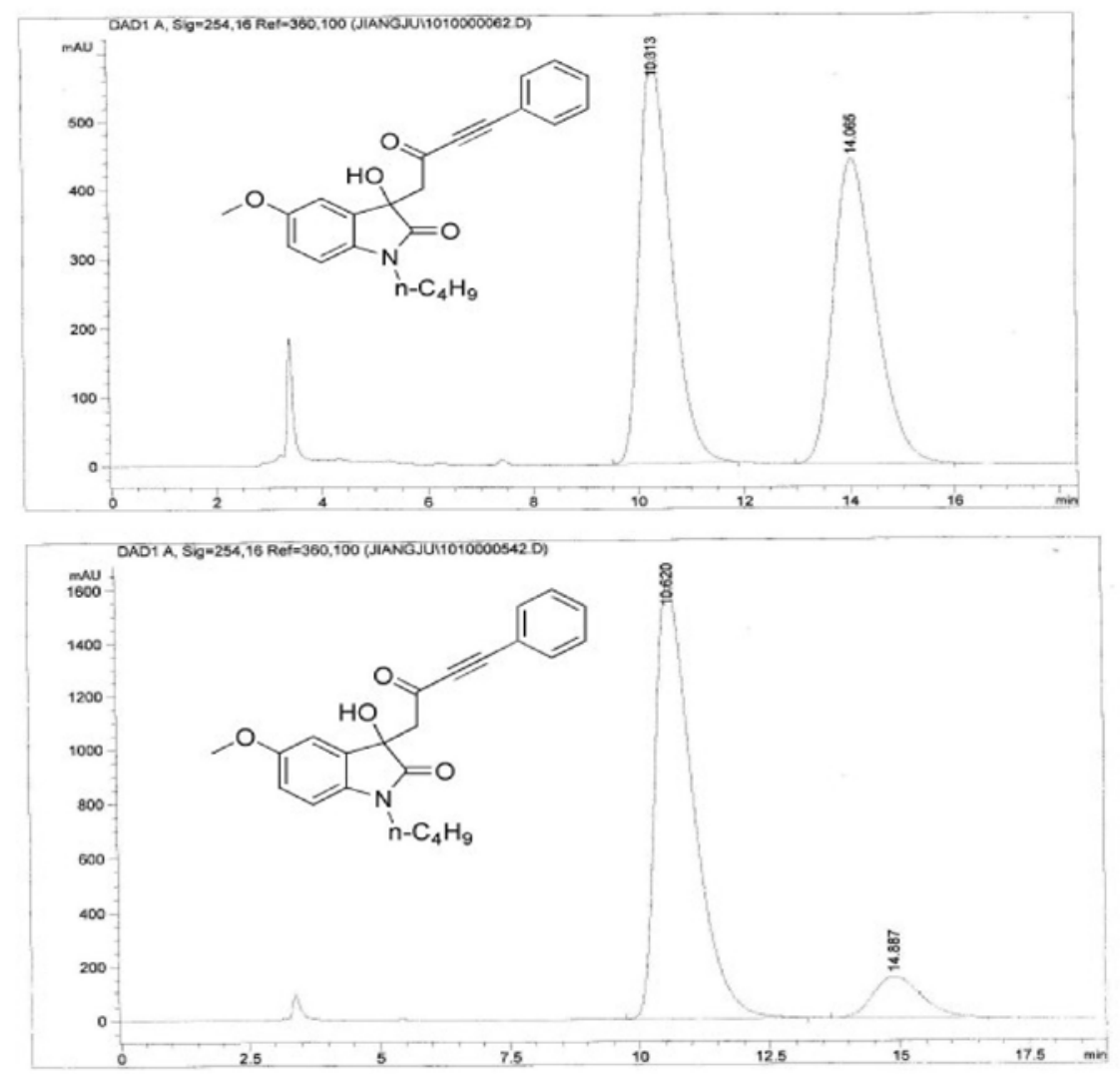

Figure S23. HPLC of compound 4i. 

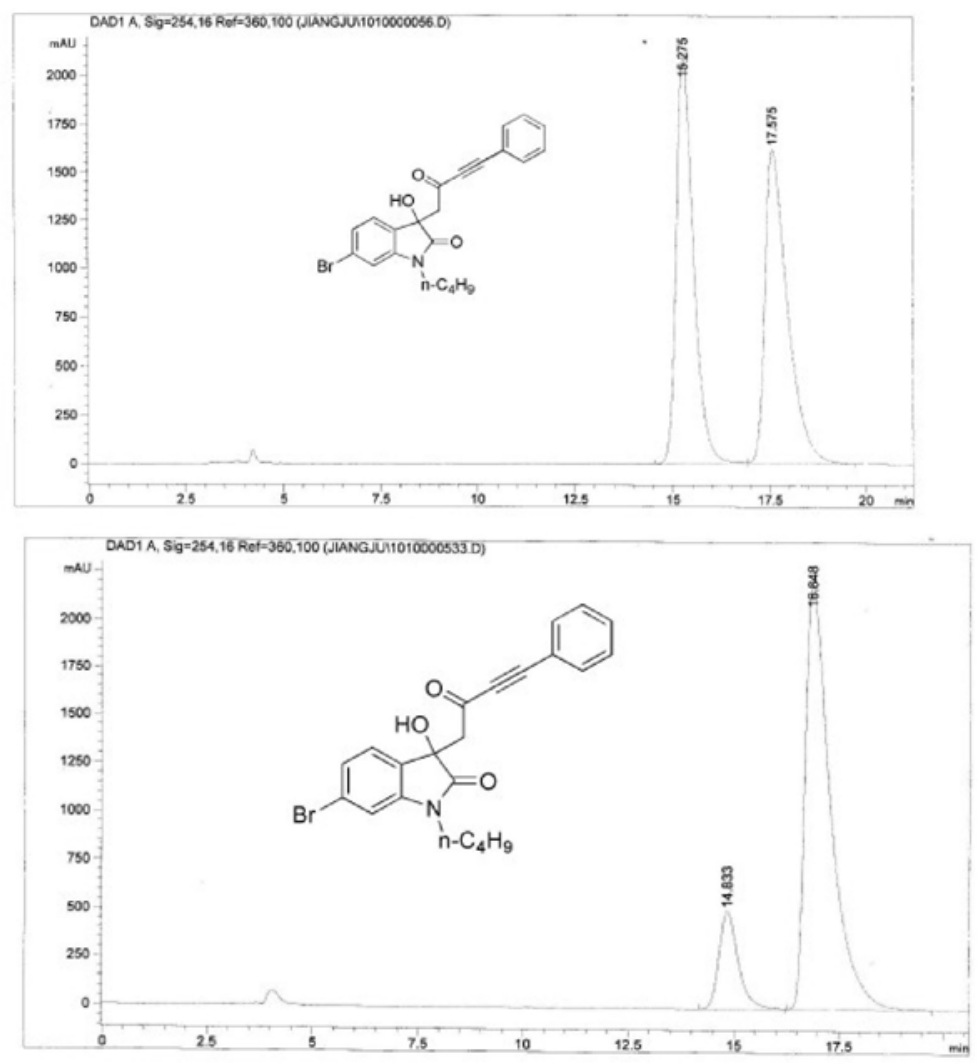

Figure S24. HPLC of compound $\mathbf{4 j}$.
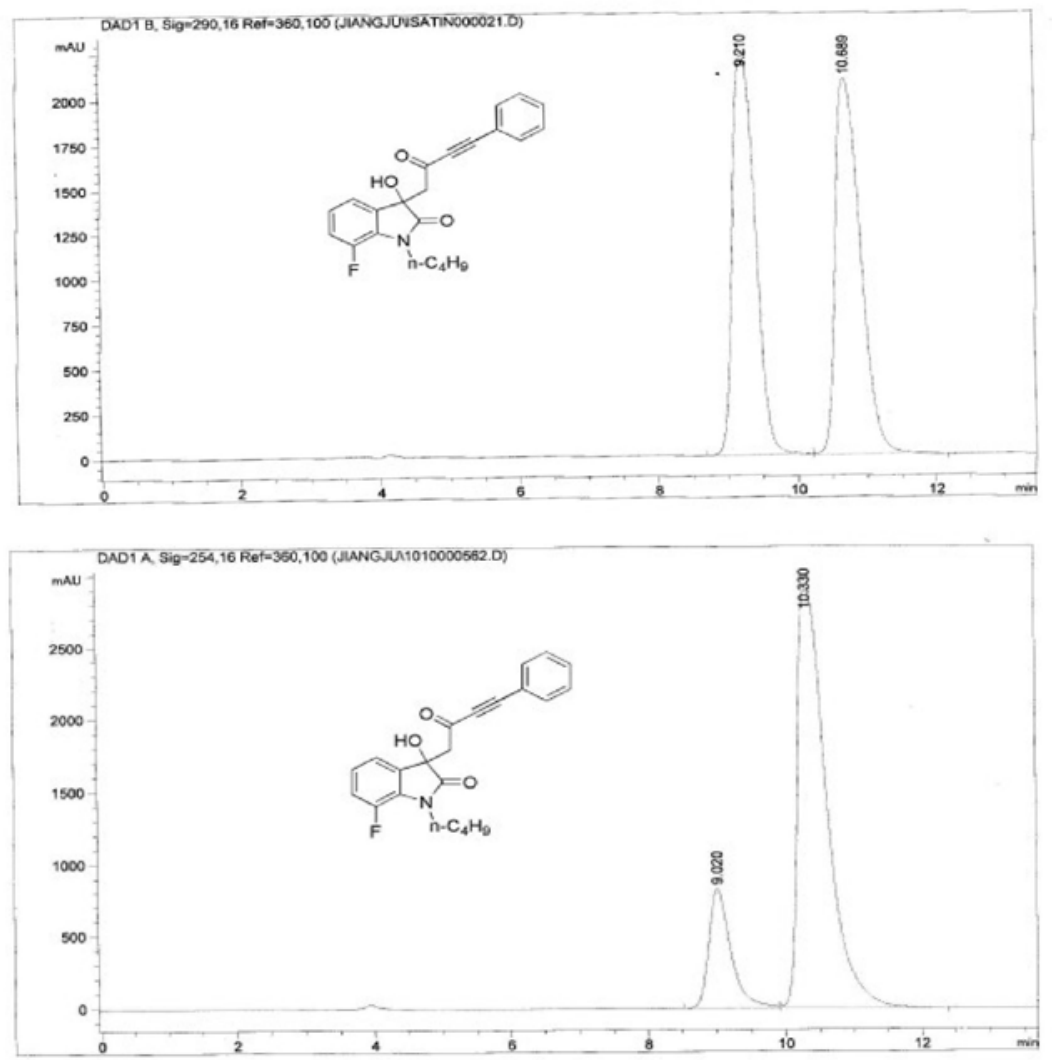

Figure S25. HPLC of compound 4k. 

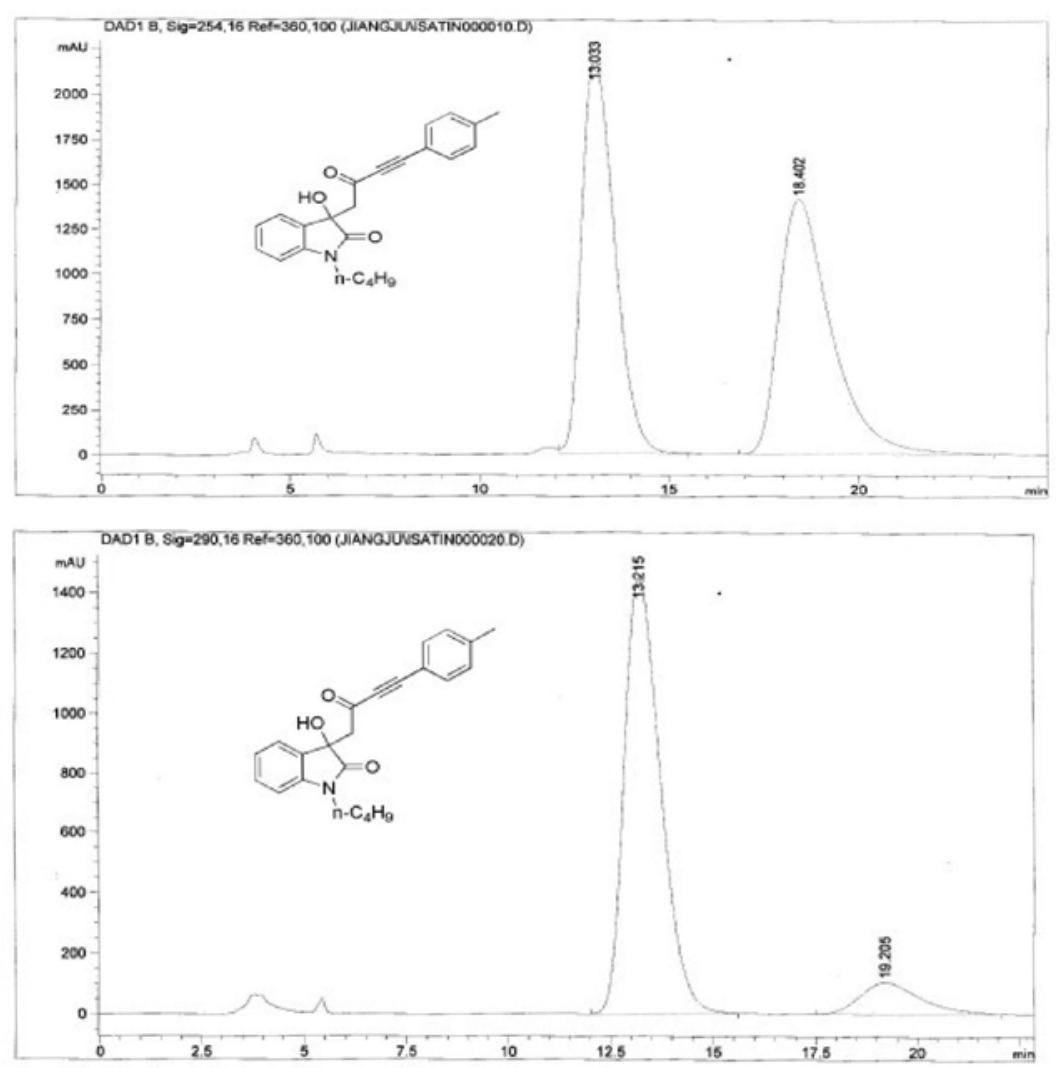

Figure S26. HPLC of compound 4ba.
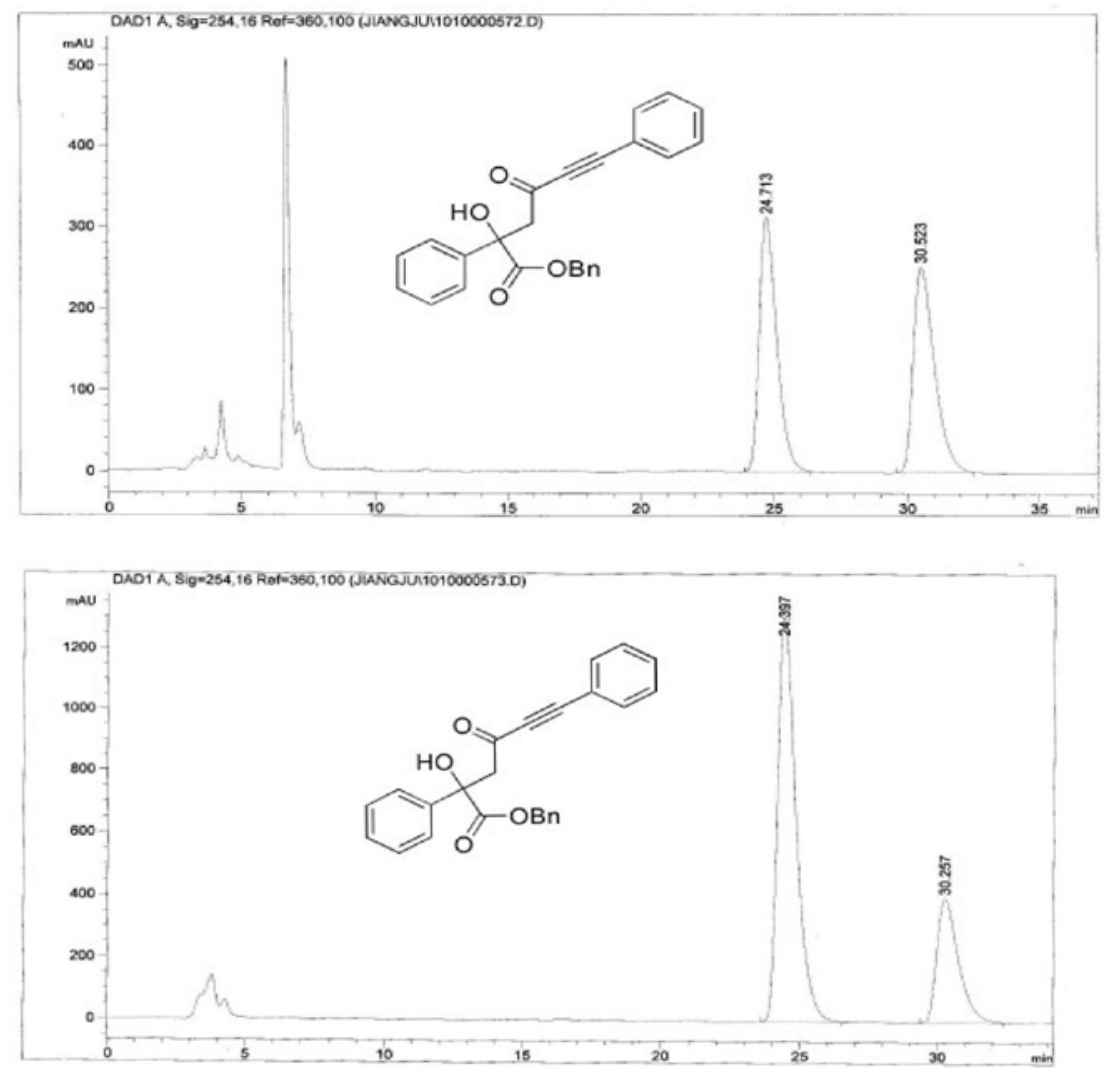

Figure S27. HPLC of compound 41. 
S26

Asymmetric Organocatalytic Synthesis of $\beta$-Hydroxyynones with a Quaternary Carbon Center

J. Braz. Chem. Soc.
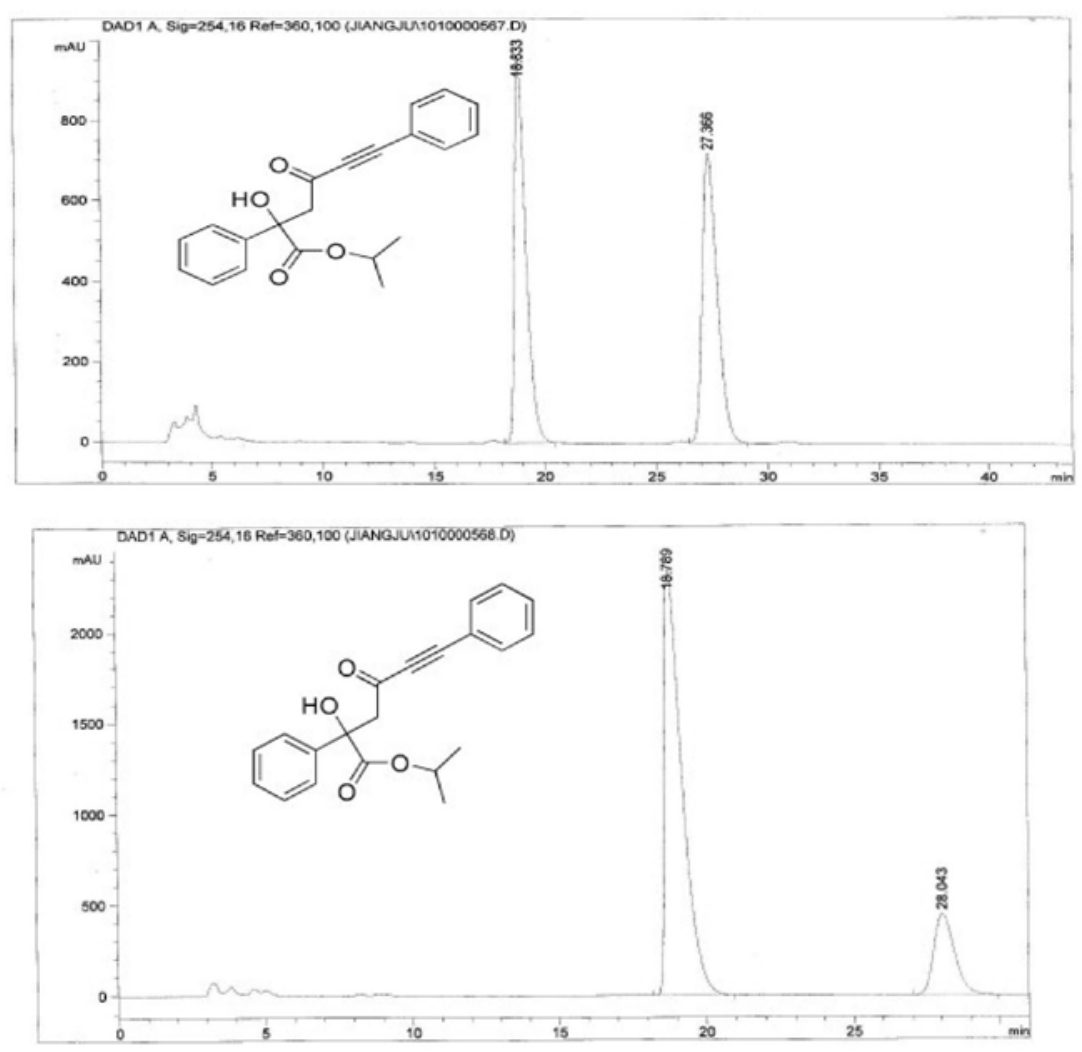

Figure S28. HPLC of compound Mm.
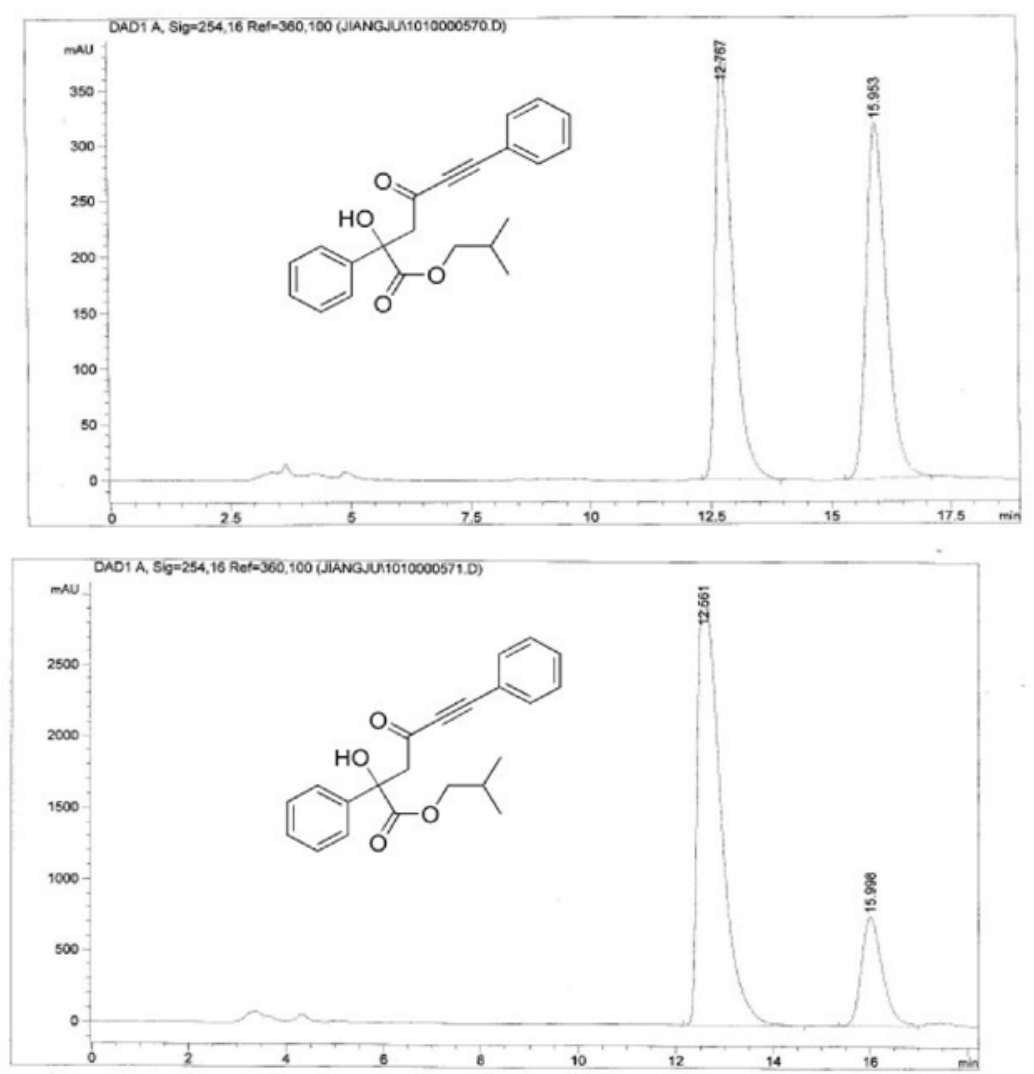

Figure S29. HPLC of compound An. 